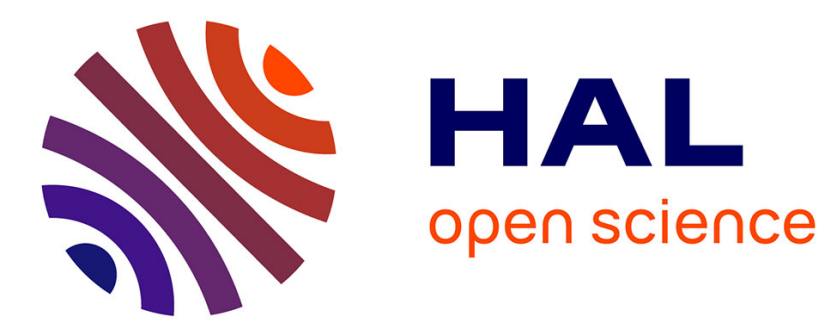

\title{
An Overview of ONERA Research Activities Related to Drag Analysis and Breakdown
}

Didier Bailly, Ilias Petropoulos, Christelle Wervaecke, Michaël Méheut, Olivier Atinault, Camille Fournis

\section{- To cite this version:}

Didier Bailly, Ilias Petropoulos, Christelle Wervaecke, Michaël Méheut, Olivier Atinault, et al.. An Overview of ONERA Research Activities Related to Drag Analysis and Breakdown. AIAA AVIATION 2021 FORUM, Aug 2021, VIRTUAL EVENT, France. 10.2514/6.2021-2551 . hal-03449322

\section{HAL Id: hal-03449322 \\ https://hal.science/hal-03449322}

Submitted on 25 Nov 2021

HAL is a multi-disciplinary open access archive for the deposit and dissemination of scientific research documents, whether they are published or not. The documents may come from teaching and research institutions in France or abroad, or from public or private research centers.
L'archive ouverte pluridisciplinaire HAL, est destinée au dépôt et à la diffusion de documents scientifiques de niveau recherche, publiés ou non, émanant des établissements d'enseignement et de recherche français ou étrangers, des laboratoires publics ou privés. 


\title{
An overview of ONERA research activities related to drag analysis and breakdown
}

\author{
Didier Bailly *, Ilias Petropoulos ${ }^{\dagger}$, Christelle Wervaecke ${ }^{\ddagger}$, Michaël Méheut ${ }^{\S}$, Olivier Atinault II , and Camille Fournis ॥ \\ ONERA - The French Aerospace Lab, Meudon, F-92190, France
}

\begin{abstract}
Drag is a design parameter of primary interest in aerodynamics performance evaluation. Its accurate prediction and phenomenological decomposition can provide a valuable physical insight into its origins, and also gives a basis for aircraft design improvements or optimization. In addition, thrust/drag bookkeeping is of primary interest in aircraft design for the decomposition of the airframe and engine contributions to overall aircraft performance. Nevertheless, for innovative configurations such as highly-integrated aero-propulsive concepts this decomposition may be difficult. The above have motivated the investigation of several theories and approaches for drag analysis and decomposition over the last century. The present paper gives an overview of ONERA methods dedicated to the analysis of drag, both from computational fluid dynamics simulations and wind tunnel experiments.
\end{abstract}

\section{Introduction}

In aeronautics, drag is one of the most important parameters evaluated during the design process. Its value has a direct impact on the performance of an aircraft: its range, its carrying capacity and above all its energy or fuel consumption. Thus, drag is not only linked to the aerodynamic performance of an airplane, but also to its environmental impact. Within a design cycle, the correct assessment and improvement of drag performance requires the development of methods that are not only accurate, but which can also provide a decomposition of drag associated to specific fluid phenomena, thus making it possible to identify, locate and quantify the influence of each such phenomenon on the total aircraft drag. This information thus guides and improves, for example, the resolution of design problems or the estimation of drag control methods.

The most straightforward way to evaluate the drag is to determine the action of the fluid on the body. In general this is called the near-field approach. In wind tunnel testing, the use of balances provides an overall assessment of the forces. Parietal pressure sensors or gauges allow a first breakdown into pressure and friction drag components. However, this is not very accurate due to the fragmentary information obtained. In the case of numerical simulations, this method consists in integrating the forces over the body surface. The two components which can be determined (pressure and friction) are the normal and tangential components of the force acting on the body, projected onto the freestream direction. A different approach consists in evaluating the effects of the body on the fluid by using the momentum conservation law. This is called the far-field approach and leads to a physical drag breakdown after some modeling considerations. In general, three components are considered: induced, wave and viscous drag associated respectively to vortex shedding past lifting bodies, shock waves and viscous phenomena.

The far-field approach was originally applied to wake survey data. In 1925, Betz was the first to propose a profile drag expression as a function of the static and stagnation pressure obtained by wake survey [1]. Jones' work furnished, in 1937, a modified and more reliable expression [2]. Later, in 1956, Oswatitsch proposed a profile drag expression as a function of the entropy in the far-field, using small disturbance assumptions [3]. In 1972, Maskell established a first drag decomposition into profile and induced drag components [4]. The induced drag is expressed from a wake survey limited to the vortical wake and can take into account or not the influence of the tunnel walls by using different adapted Green functions. In the 2000s, Kusunose published a complete drag decomposition method with the three components for compressible flows [5-7]. In particular, the decomposition of the profile drag in its wave and viscous components is obtained by using the vorticity field of the wake. This means that it is mainly dedicated to three dimensional flows.

\footnotetext{
* Research Scientist, Aerodynamics, Aeroelasticity and Acoustics Department, didier.bailly@onera.fr

${ }^{\dagger}$ Research Scientist, Aerodynamics, Aeroelasticity and Acoustics Department, ilias.petropoulos@onera.fr

$\doteqdot$ Research Scientist, Aerodynamics, Aeroelasticity and Acoustics Department, christelle.wervaecke@onera.fr

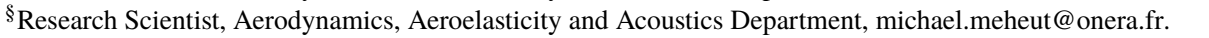

IResearch Scientist, Aerodynamics, Aeroelasticity and Acoustics Department, christelle.wervaecke@onera.fr

" Ph.D. Student, Aerodynamics, Aeroelasticity and Acoustics Department, camille.fournis@onera.fr
} 
In 1972, Steger and Baldwin developed a method for the extraction of wave drag from a full potential solution [8]. They showed that the gain in momentum across the shock makes it possible to calculate the wave drag thanks to an integration on an area surrounding the shock. The integration avoiding the leading edge, where the numerical errors are the highest, the results must be more accurate than those obtained by an integration of the pressure at the skin. This will be confirmed by the spurious drag evaluation techniques. In their paper, the authors present a far-field formulation, generalizing that of Oswatitsch, but limited to iso-energetic flows. In 1978, Henne and Hicks presented a far-field induced drag formulation [9]. Then other authors have also studied drag prediction for supersonic and unsteady flows.

In 1983, Yu applied Oswatitsch's expression to numerical solutions of the Euler equations in order to determine the wave drag and noted a dependence of the latter on the integration contour [10]. The spurious drag phenomenon is again found to influence the accuracy of results. This notion was clearly introduced by Lock in 1985 [11] and Rizzi [12] the same year. It was then further illustrated in the paper of Destarac in 1993, paper in which the use of physical sensors is introduced allowing the definition of integration volumes in order to exclude spurious phenomena [13]. Induced drag was studied by the group of Van Dam starting from 1991, inspired by Maskell's formulation [14, 15]. Again, numerical errors lead to a dependence on the integration domain.

For Navier-Stokes results, the method is similar to that of the Euler results. In 1990, van der Vooren and Slooff provided all the theoretical elements allowing for a far-field extraction [16]. In 2000, drag decomposition results are presented by Tognaccini and Paparone [17]. Finally, in 2004, a formulation applicable to power-on flows is presented by van der Vooren and Destarac [18].

For many years, ONERA has been working on the development of drag extraction methods from both experimental and numerical data. To study numerical simulations, the FFD family of codes has been developed from the van der Vooren approach. The initial version was devoted to steady flows, with or without power-on conditions. Many theoretical improvements have since been added, such as the evaluation of the spurious drag and the viscous pressure drag definition. Thereafter, the field of application was extended to rotor flows (helicopter or propeller) by generalizing the van der Vooren approach to rotating reference frames, and finally to unsteady flows. However the formulation is based on thermodynamic considerations where entropy and enthalpy variations are the essential parameters. The formulation obtained thus explicitly defines terms such as viscous and wave drag due to irreversible processes. The induced drag does not have a direct definition. It is rather defined as the remainder of the total drag from which the irreversible terms have been subtracted. This is the reason why work is currently being carried out to develop a complementary formulation which is primarily based on the velocity vector field (and in particular the Lamb vector). Last, alternative methods for aerodynamic performance evaluation are investigated, in particular based on exergy analysis. In parallel, methods for experimental drag evaluation and decomposition have been developed based on five-hole probe or Particle Image Velocimetry (PIV) measurements.

\begin{tabular}{lllll}
\hline \hline \multicolumn{2}{c}{ CFD } & & \multicolumn{2}{c}{ Wind tunnel } \\
\hline FFD & Lamb vector & Exergy & Five-hole probes & PIV \\
$\bullet$ steady/unsteady & $\bullet$ steady & $\bullet$ steady & $\bullet$ steady & $\bullet$ steady \\
$\bullet$ sub/transonic & $\bullet$ sub/transonic & $\bullet$ sub/transonic & $\bullet$ sub/transonic & $\bullet$ subsonic \\
$\bullet$ power off/on & & $\bullet$ power off/on & \\
$\bullet$ rotating frame & & & \\
\hline \hline
\end{tabular}

Table 1 Overview of the domain of application of ONERA methods related to drag analysis.

An overview of methods developed at ONERA and their domain of application is shown in Table 1 . These are presented in further detail in the main part of the paper. Section II focuses on methods for drag extraction and decomposition from numerical solutions. Then, Section III presents methods developed for the analysis of drag in wind tunnel experiments. 


\section{CFD}

\section{A. The van der Vooren/Destarac formulation}

\section{The van der Vooren formulation}

This far-field method is derived from the steady form of the continuity and the Navier-Stokes momentum equations applied to a control volume $\Omega$ surrounding the aircraft. By considering only the surface forces on the body:

$$
\int_{\partial \Omega}\left(\mathbf{q}-\mathbf{q}_{\infty}\right) \rho \mathbf{q} \cdot \mathbf{n} d S=-\int_{\partial \Omega}\left(p-p_{\infty}\right) \mathbf{n} d S+\int_{\partial \Omega} \tau . \mathbf{n} d S
$$

where $\rho, \mathbf{q}, p, \tau$ and $\mathbf{n}$ are respectively the density, the velocity vector, the pressure, the viscous stress tensor in the fluid and the outwards-pointing unit normal vector on the integration surface. The subscript $\infty$ refers to the freestream state of the fluid. The surface $\partial \Omega$ is split into two surfaces $S_{A}$ and $S_{e}$, respectively the aircraft and exterior surfaces and the equation (1) is projected along the free-stream direction (x-coordinate) to give:

$$
\int_{S_{e}}\left(u-u_{\infty}\right) \rho \mathbf{q} \cdot \mathbf{n} d S=-\int_{S_{e} \cup S_{A}}\left(p-p_{\infty}\right) \mathbf{i} \cdot \mathbf{n} d S+\int_{S_{e} \cup S_{A}} \text { i. } \tau \cdot \mathbf{n} d S
$$

where $u$ is the velocity component in the x-direction. The near-field drag $\left(D_{n f}\right)$ is the sum of the pressure drag $\left(D_{p}\right)$ and the friction drag $\left(D_{f}\right)$ given by:

$$
D_{n f}=D_{p}+D_{f}=\int_{S_{A}}\left(p-p_{\infty}\right) \mathbf{i} . \mathbf{n} d S-\int_{S_{A}} \mathbf{i} \cdot \tau \cdot \mathbf{n} d S
$$

A far-field drag expression is obtained from the two equations (2) and (3):

$$
D_{f f}=-\int_{S_{e}}\left[\left(u-u_{\infty}\right) \rho \mathbf{q}+\left(p-p_{\infty}\right) \mathbf{i}-\mathbf{i} \cdot \tau\right] \cdot \mathbf{n} d S
$$

This equation ensures an exact balance between these two expressions, $D_{n f}=D_{f f}$, but gives no information about a physical breakdown.

The formulation developed at ONERA is based on the van der Vooren formulation, built upon the distinction between reversible and irreversible thermodynamic processes. The first step is to suppose a flow without trailing vorticity and to consider the velocity far downstream where $p=p_{\infty}$. In this case, the velocity is formulated as:

$$
\bar{u}=u_{\infty} \sqrt{1+\frac{2 \Delta H}{u_{\infty}^{2}}-\frac{2}{\gamma-1} \frac{1}{M_{\infty}^{2}}\left(e^{\Delta s / C_{p}}-1\right)}
$$

where $\Delta H$ and $\Delta s$ are the enthalpy and entropy perturbations and $M_{\infty}$ is the freestream Mach number. The quantity under the square root is positive only if the local stagnation pressure is higher than the free-stream static pressure, $p_{i}>p_{\infty}$. It can been shown that $\bar{u}$ may be undefined downstream of a sufficiently strong shock wave and in the core of a vortex. Furthermore, it can be shown that $\bar{u}$ is not defined inside the boundary layer very close to the wall if the Mach number outside the boundary layer is locally greater than $M_{\infty}$. So for each drag source, there may exist a domain where $\bar{u}$ is not defined.

Under these assumptions, the far-field drag takes the form:

$$
D_{f f}=-\int_{S_{e}}\left(\bar{u}-u_{\infty}\right) \rho \mathbf{q} \cdot \mathbf{n} d S=-\int_{S_{e}} \Delta \bar{u} \rho \mathbf{q} \cdot \mathbf{n} d S
$$

With the classical notations, the vector $\mathbf{f}$ is defined and decomposed in two components such that:

$$
\mathbf{f}=\mathbf{f}_{\mathrm{vw}}^{*}+\mathbf{f}_{\mathbf{i}}^{*}=-\rho\left(u-u_{\infty}\right) \mathbf{q}-\left(p-p_{\infty}\right) \mathbf{i}+\mathbf{i} \cdot \tau
$$

with

$$
\left\{\begin{array}{l}
\mathbf{f}_{\mathbf{v w}}^{*}=-\Delta \bar{u} \rho \mathbf{q}+\mathbf{i} \cdot \tau \\
\mathbf{f}_{\mathbf{i}}^{*}=-\rho\left(u-u_{\infty}-\Delta \bar{u}\right) \mathbf{q}-\left(p-p_{\infty}\right) \mathbf{i}
\end{array}\right.
$$


The second step is to decompose the control volume and make the assumption that viscous phenomena and shock waves are confined within specific volumes. Outside these volumes the flow is supposed inviscid. The irreversible drag components are then defined as:

$$
D_{v}+D_{w}=\int_{S_{v}} \mathbf{f}_{\mathrm{vw}}^{*} \cdot \mathbf{n} d S+\int_{S_{w}} \mathbf{f}_{\mathbf{v w}}^{*} \cdot \mathbf{n} d S
$$

The induced drag is then:

$$
D_{i}=\int_{S_{v} \cup S_{w}} \mathbf{f}_{\mathbf{i}}^{*} \cdot \mathbf{n} d S
$$

This ensures an exact near-field / far-field drag balance:

$$
D_{n f}=D_{f f}=D_{p}+D_{f}=D_{v}+D_{w}+D_{i}
$$

The advantages of the van der Vooren formulation are the exact drag balance and the possibility to extend it to power-on configurations. To integrate this theoretical formulation in a computational code, an adaptation is required in order to maintain high accuracy results from numerical simulation data. The following two sections introduce two essential concepts for such an integration.

The one-vector formulation It is possible to express all the far-field drag components with $\mathbf{f}_{\mathbf{i}}^{*}$ alone, by an elimination of $\mathbf{f}_{\mathbf{v w}}^{*}$ from the viscous and wave drag components [19]. Due to the zero divergence property of the vector $\mathbf{f}$, the wave drag can be expressed as follows:

$$
D_{w}=\int_{S_{w}} \mathbf{f}_{\mathbf{v w}}^{*} \cdot \mathbf{n} d S=-\int_{S_{w}} \mathbf{f}_{\mathbf{i}}^{*} \cdot \mathbf{n} d S
$$

Furthermore, even though the vectors $\mathbf{f}_{\mathrm{vw}}^{*}$ and $\mathbf{f}_{\mathbf{i}}^{*}$ may not be defined inside all boundary layer regions, the wall boundary condition allows them to be numerically defined as:

$$
\left\{\begin{array}{l}
D_{f}=-\int_{S_{A}} \mathbf{f}_{\mathbf{v w}}^{*} \cdot \mathbf{n} d S \\
D_{p}=-\int_{S_{A}} \mathbf{f}_{\mathbf{i}}^{*} \cdot \mathbf{n} d S
\end{array}\right.
$$

While by introducing the viscous pressure $\operatorname{drag} D_{v p}$ :

$$
D_{v p}=D_{v}-D_{f}=D_{p}-\int_{S_{v}} \mathbf{f}_{\mathbf{i}}^{*} \cdot \mathbf{n} d S
$$

These results give the basic breakdown of the pressure drag:

$$
D_{p}=D_{v p}+D_{w}+D_{i}
$$

which is equivalent to the relation (11). The motivation to adopt this formulation is essentially numerical. The computations with $\mathbf{f}_{\mathbf{i}}^{*}$ are easier and preserve a high accuracy. Also, the computation of $\tau$ is only limited to the total drag breakdown.

Spurious drag For numerical simulations, very early studies have identified a source of error which reduces the precision of results, thus making them deviate from the expected theoretical results. This phenomenon, commonly referred to as spurious drag production, originates from numerical mechanisms related to the techniques used to solve the flow equations. The source is the production of entropy due to truncation errors, numerical errors and artificial dissipation techniques. It may thus depend at the same time on the properties of the computational mesh, the numerical scheme and the level of convergence. It should be noted that this phenomenon is purely numerical and affects both the far-field and the near-field methods. However, far-field methods analyze the entire flow and allow the quantification of an important part of spurious drag, unlike their near-field counterparts. A study of an airfoil in inviscid flow shows the main location of the production of spurious entropy to be at the leading and trailing edges and near the shocks, if they are present [13]. The influence of the properties of the mesh and of the artificial dissipation can be quantified with a far-field analysis by an appropriate construction of integration volumes. RANS studies show similar mechanisms [19]. In the same study, the author shows that spurious entropy production is also responsible for the decay of wake vortices by a process of numerical diffusion. The mesh influences the accuracy of results due to its refinement but also its extension. 
So, the numerical dissipation affects both irreversible and reversible drag components. The theoretical balance equation (11) was written assuming that irreversible phenomena are confined within the volumes $V_{v}$ and $V_{w}$ and neglecting the numerical effects. These can in turn be evaluated by defining a spurious drag equivalent to an irreversible drag but in the volume $V_{s p}=\Omega \backslash\left(V_{v} \cup V_{w}\right)$ :

$$
D_{s p}=\int_{\partial V_{s p}} \mathbf{f}_{\mathrm{vw}}^{*} \cdot \mathbf{n} d S
$$

This reforms the balance equations as follows:

$$
\left\{\begin{array}{c}
D_{p}+D_{f}=D_{v}+D_{w}+D_{i}+D_{s p} \\
D_{p}=D_{v p}+D_{w}+D_{i}+D_{s p}
\end{array}\right.
$$

Further detailed investigations have demonstrated the complexity of the spurious drag concept [20-22]. It has been shown that spurious pressure drag can be broken down into three components produced by three distinct numerical phenomena. The first component is of irreversible nature and linked primarily to spurious entropy production and vorticity in the direction normal to the velocity. The second, also of irreversible nature, is related to the spurious destruction of vorticity parallel to the velocity. The third component is of reversible nature and is a pure product of the far-field boundary condition.

\section{Formulation extensions to a rotating reference frame}

At a first step, the formulation described above has been extended to steady flows in a rotating frame of reference [23, 24]. An adaptation of the method to a rotor in hover leads to the consideration of rotor torque instead of aircraft drag, which gives the decomposition into the three terms: wave, viscous and induced torque. Rotor simulations differ from aircraft ones inasmuch as the equations are usually not solved in the same reference frame: aircraft simulations use the absolute velocity formulation in a fixed reference frame, whereas rotor simulations may use either the absolute or the relative velocity projected in a relative reference frame. This change of reference frame also implies some changes in the thermodynamical quantities used, in particular the use of the rothalpy $(I)$ instead of the stagnation enthalpy to determine the torque due to irreversible phenomena. At a second step, this formulation has been extended to propellers and counter-rotating open rotor configurations, enabling the extraction of the different far-field thrust and torque components [25].

This new formulation dedicated to rotating frame leads to the definition of a new tensor $\overline{\overline{f_{i}^{*}}}$ :

$$
\overline{\overline{f_{i}^{*}}}=-\rho\left(\mathbf{q}-\mathbf{q}_{\infty}-\Delta \overline{\mathbf{W}}\right) \otimes(\mathbf{q}-\mathbf{s})+\left(p-p_{\infty}\right) \overline{\bar{I}}
$$

where $\Delta \bar{W}$ is a fictive deficit of velocity from the freestream state:

$$
\Delta \bar{W}=\sqrt{2\left(\Delta I+\frac{a_{\infty}^{2}}{\gamma-1}\left(1-e^{\Delta s / C_{p}}\right)\right)+\left|\mathbf{W}_{\infty}\right|^{2}}-\left|\mathbf{W}_{\infty}\right|^{2}
$$

$\mathbf{W}_{\infty}=\mathbf{q}_{\infty}-\mathbf{s}$ defines the first axis of local aerodynamic frame around the blade for a given radius $r$ (Fig. 1), $a$ denotes the speed of sound and $\mathbf{s}$ is the absolute grid velocity vector.

The $\overline{\overline{f_{i}^{*}}}$ tensor is then used to compute the different Thrust $(T)$ and Torque $(C)$ far-field components in a similar way as in a fixed reference frame:

$$
\begin{aligned}
& T_{v}=T-\int_{S_{v}}\left(\overline{\overline{f_{i}^{*}}} \cdot \mathbf{e}_{\mathbf{x}}\right) \cdot \mathbf{n} d S \quad T_{w}=-\int_{S_{w}}\left(\overline{\overline{f_{i}^{*}}} \cdot \mathbf{e}_{\mathbf{x}}\right) \cdot \mathbf{n} d S \quad T_{i}=\int_{S_{i}}\left(\overline{\overline{f_{i}^{*}}} \cdot \mathbf{e}_{\mathbf{x}}\right) \cdot \mathbf{n} d S \\
& \underbrace{C_{v}=C-\int_{S_{v}}\left(\overline{\overline{f_{i}^{*}}} \cdot \mathbf{e}_{\theta}\right) \cdot \mathbf{n} r d S}_{\text {Viscous }} \quad \underbrace{C_{w}=-\int_{S_{w}}\left(\overline{\overline{f_{i}^{*}}} \cdot \mathbf{e}_{\theta}\right) \cdot \mathbf{n} r d S}_{\text {Wave }} \quad \underbrace{C_{i}=\int_{S_{i}}\left(\overline{\overline{f_{i}^{*}}} \cdot \mathbf{e}_{\theta}\right) \cdot \mathbf{n} r d S}_{\text {Induced }}
\end{aligned}
$$

The exact near-field/far-field balance of this new formulation allows to write:

$$
\left\{\begin{array}{l}
T=T_{p}+T_{f}=T_{v}+T_{w}+T_{s p}+T_{i L}+T_{i D} \\
C=C_{p}+C_{f}=C_{v}+C_{w}+C_{s p}+C_{i L}+C_{i D}
\end{array}\right.
$$




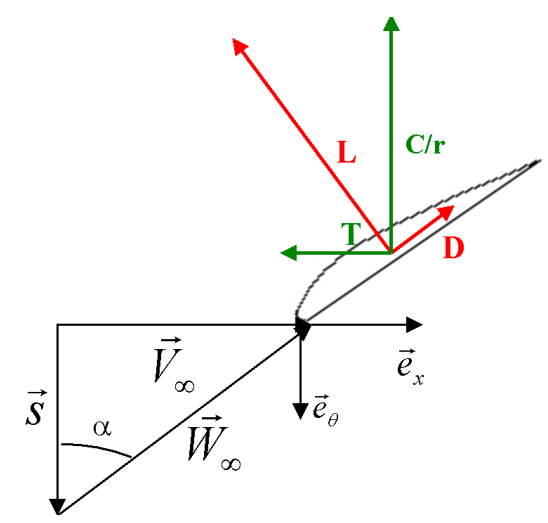

Fig. 1 Schematic view of the local aerodynamic frame around the blade at a fixed radius $r$ [25].

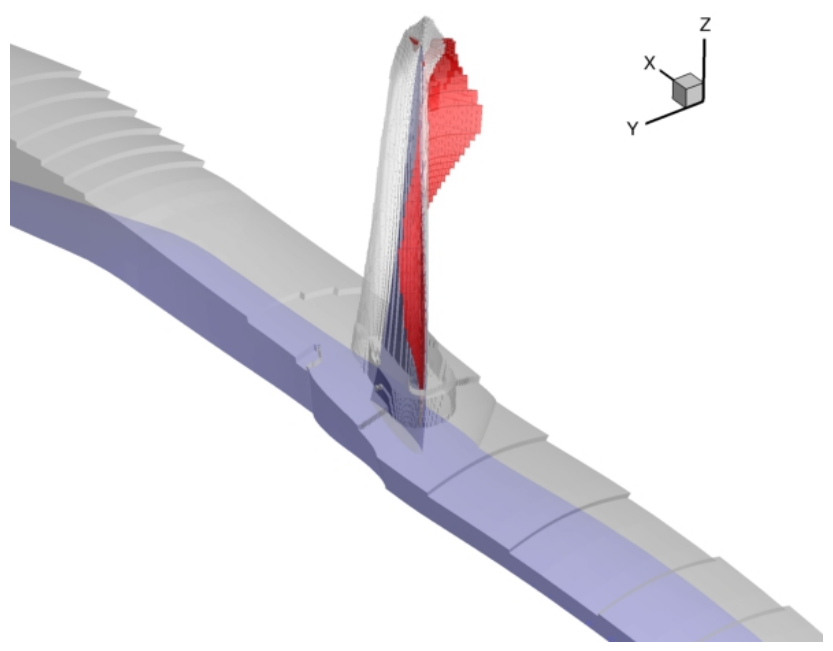

Fig. 2 AIPX7 propeller configuration at cruise conditions - Viscous (in grey) and wave (in red) control volumes [25].

with:

$$
\left\{\begin{array}{l}
T_{i D}=T_{i}-T_{i L} \\
C_{i D}=C_{i}-C_{i L}
\end{array}\right.
$$

The subscripts $L$ and $D$ in the previous equations translate, respectively, the lift and the drag contributions to the different near-field and far-field components (depending on $r$ ). In the case of a propeller made up of lifting profiles, this new breakdown of the reversible terms enables to obtain a thrust component which can be assimilated to the induced drag of the propeller $\left(T_{i D}\right)$ and a component which represents a "net thrust" which is only due to the lift of the different profiles of the blades $\left(T_{i L}\right)$.

Application to an isolated propeller configuration This formulation was applied to the isolated AIPX7 propeller configuration (designed by Airbus and made up of 11 blades) in order to understand the physical meaning of the different far-field components and determine the respective contributions of each component in cruise flight conditions $\left(M_{\infty}=0.73\right)$. The computations were carried out using the elsA CFD solver (ONERA-Airbus-Safran property) [26]. Fig. 2 shows the viscous and wave volumes defined for this configuration using the in-house far-field drag extraction software.

As far as the far-field breakdown is concerned, Table 2 presents the contribution of the different components to the total far-field thrust and torque. In these transonic conditions, the wave components are not zero and are respectively equal to $0.9 \%$ and $2.3 \%$ of the far-field drag thrust and torque. The viscous components are respectively equal to $44.8 \%$ 


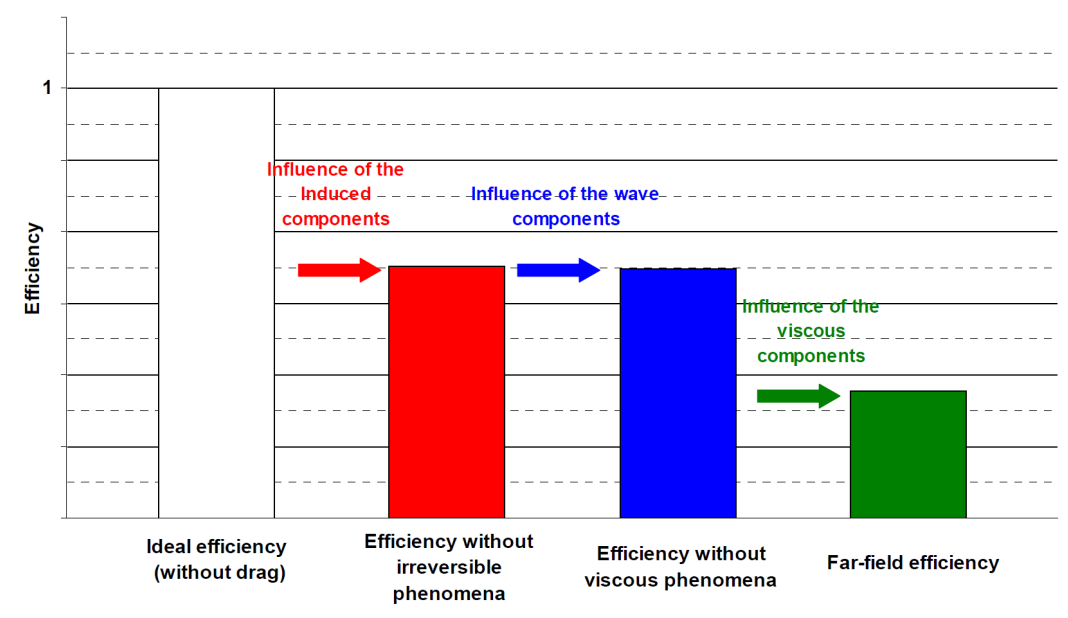

Fig. 3 AIPX7 propeller configuration at cruise conditions - Evolution of the efficiency with far-field components [25].

and $33.0 \%$ of the far-field drag thrust and torque.

The lift thrust force which corresponds to the force allowing the forward motion of the propeller, is 3.4 times higher than the total far-field drag. For the torque, the lift term, which also faces up to the motion of the blade, is largely higher than the far-field drag components. This far-field breakdown also enables to identify the spurious thrust and torque that slightly modifies the efficiency of the propeller compared to the near-field approach.

\begin{tabular}{cccccccc}
\hline \hline & Viscous & Wave & Induced & Far-field drag & Lift & Total far-field & Spurious \\
\hline Thrust & $-18.8 \%$ & $-0.4 \%$ & $-22.8 \%$ & $-42.0 \%$ & $142.0 \%$ & $100.0 \%$ & $0.4 \%$ \\
Torque & $1.4 \%$ & $0.1 \%$ & $4.4 \%$ & $5.9 \%$ & $94.1 \%$ & $100.0 \%$ & $0.1 \%$ \\
\hline \hline
\end{tabular}

Table 2 AIPX7 propeller configuration at cruise conditions - Far-field breakdown [25].

Fig. 3 shows the impact of the different far-field thrust and torque terms on the propeller efficiency, computed using the following equation:

$$
E_{f}=\left|\frac{T u_{\infty}}{C \omega}\right|
$$

The ideal behavior, with an efficiency of 1 , is obtained by assuming that the drag components are zero. In these cruise conditions, the reduction of the efficiency is mainly due to the induced and viscous phenomena, while transonic effects remain lower.

\section{Formulation extension for unsteady flows}

A new study has then led to the development of a far-field drag prediction method aiming at a phenomenological breakdown of drag for unsteady flows. A first attempt was made by Gariepy et al. [27], but the formulation developed was very sensitive to the volumes of integration and some terms were not directly linked to physical phenomena. The first step at ONERA [28] has consisted in generalizing the steady formulation of van der Vooren to unsteady flows. The demonstration relies on considering each irreversible drag source, such as shock waves and boundary layers, isolated and working in the streamtubes enclosing them. This allows to express unsteady wave and viscous drag coefficients defined in distinct volumes. The complementary part of the total unsteady drag was then defined as an unsteady induced drag, where the complementary surfaces and volumes are defined as $S_{c d}=S_{d} \backslash\left(S_{w d} \cup S_{v d}\right)$ and $V_{c}=V \backslash\left(V_{w} \cup V_{w d} \cup V_{v}\right)$ (Fig. 4).

First test cases have led to the definition of a contribution due to acoustics in the unsteady induced drag coefficient [30, 31]. To do so, the volume term of the unsteady induced drag component has been broken into two 


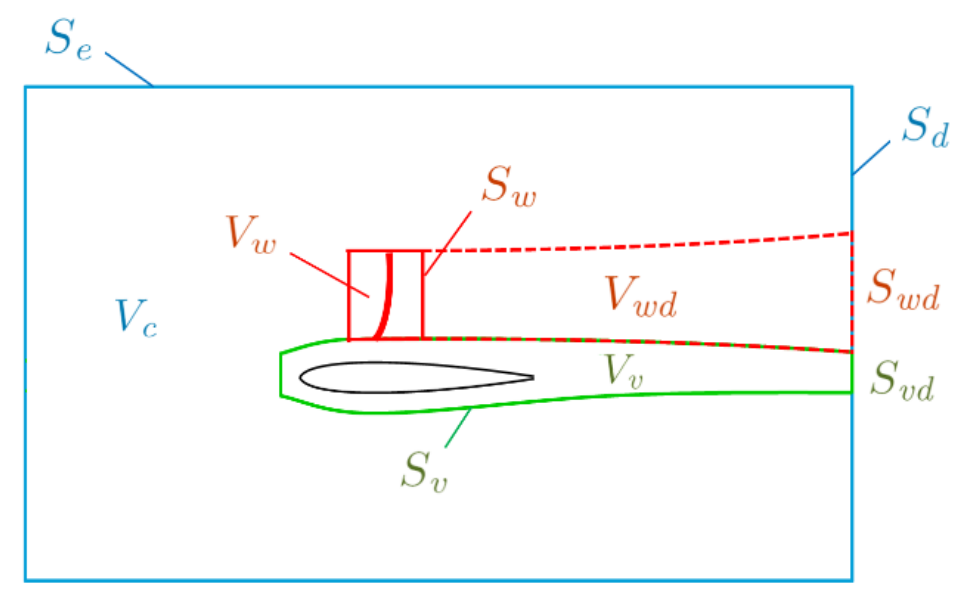

Fig. 4 Integration volumes and surfaces [29].

components, using the definition of the Riemann invariant $W^{+}=u+\frac{2 a}{\gamma-1}$ and assuming a potential flow, which is a valid assumption in the complementary volume. The final unsteady formulation is therefore:

$$
D_{f f}=D_{w}+D_{v}+D_{i}+D_{p a}+D_{m}
$$

with

$$
\begin{aligned}
& D_{w}=-\int_{S_{w}} \rho \Delta \bar{u} \mathbf{q} \cdot \mathbf{n} d S-\int_{V_{w}} \frac{\partial \rho\left(u-u_{\infty}\right)}{\partial t} d V+\int_{V_{w d}}\left(\frac{\partial \rho(\bar{u}-u)}{\partial t}-\frac{1}{\bar{u}} \frac{\partial p}{\partial t}\right) d V \\
& D_{v}=-\int_{S_{v}}\left(\rho \Delta \bar{u} \mathbf{q}-\tau_{\mathbf{x}}\right) \cdot \mathbf{n} d S-\int_{V_{v}} \frac{\partial \rho\left(u-u_{\infty}\right)}{\partial t} d V \\
& D_{i}=-\int_{S_{e}}\left[\rho(u-\bar{u}) \mathbf{q}+\left(p-p_{\infty}\right) \mathbf{i}\right] \cdot \mathbf{n} d S-\int_{V_{c}} \rho(u-\bar{u})\left(\frac{1}{a}-\frac{1}{\bar{u}}\right) \frac{\partial u}{\partial t} d V \\
& D_{p a}=-\int_{V_{c}} \rho \frac{u-\bar{u}}{a} \frac{\partial W^{+}}{\partial t} d V \\
& D_{m}=-\int_{S_{a}} \rho\left(u-u_{\infty}\right) \mathbf{q} \cdot \mathbf{n} d S
\end{aligned}
$$

Axes for the improvement of the robustness and physical background have then been explored. Acoustic contributions have in particular been highlighted and quantified. The resulting five-component formulation has then been applied to simple cases, in order to validate the phenomenological breakdown as best as possible. In these applications, the behavior of the drag components proved to be consistent with the physics of the flow. Finally, the method has been applied to complex cases in order to demonstrate its capabilities: a 3-D pitching wing case and a transonic buffet flow simulated by the Zonal Detached Eddy Simulation (ZDES) method [29, 32]. The last case is the transonic buffet over the supercritical OAT15A profile with chord $0.23 \mathrm{~m}$ at $M_{\infty}=0.73, \alpha=3.5^{\circ}$ and $R e=3.10^{6}$. A visualization (Fig. 5) of the flow field is shown at the time step when the shock wave is at the most upstream position and a strong separation occurs. The turbulent structures are depicted in Fig. 5a

URANS simulations have also been performed in a previous work. The test case was also an OAT15A profile at $M_{\infty}=0.73$ but the angle of attack was larger, $\alpha=4.5^{\circ}$, since these URANS simulations fail to predict buffet at smaller angles of attack. Furthermore, the physics simulated in the boundary layer by both models is completely different. The results can therefore only be compared qualitatively (Fig. 6). The general trends are quite similar in terms of phase between the components and with the motion of the shock wave. The viscous drag coefficient in particular is especially similar. The average wave drag is smaller in the ZDES case, which is consistent with the angle of attack being smaller. The amplitude of oscillation is also slightly smaller. The induced drag has a similar behavior in both cases, although it has stronger peaks in the ZDES case (it was constant and almost zero in the URANS case). 


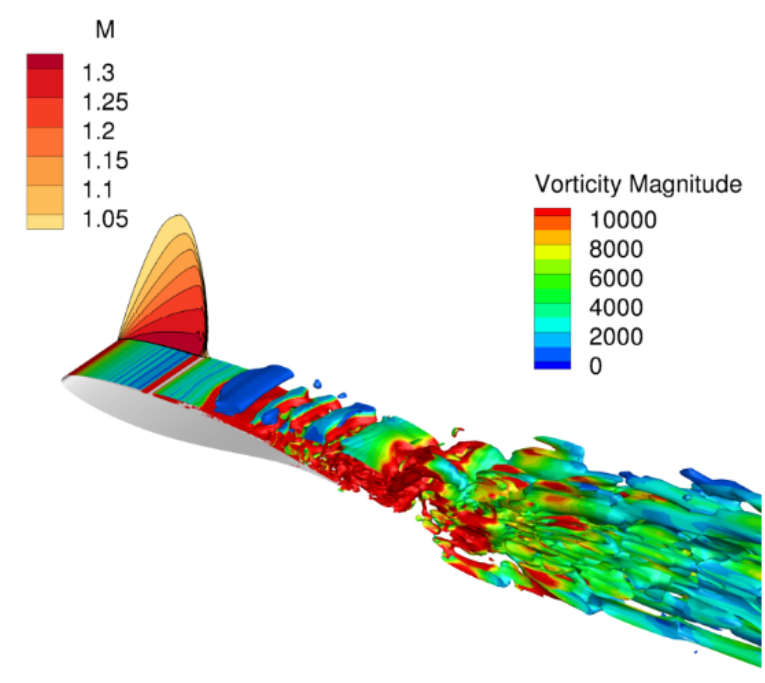

(a) Isosurfaces of the Q-criterion colored by the vorticity magnitude, and Mach contours in a transverse plane

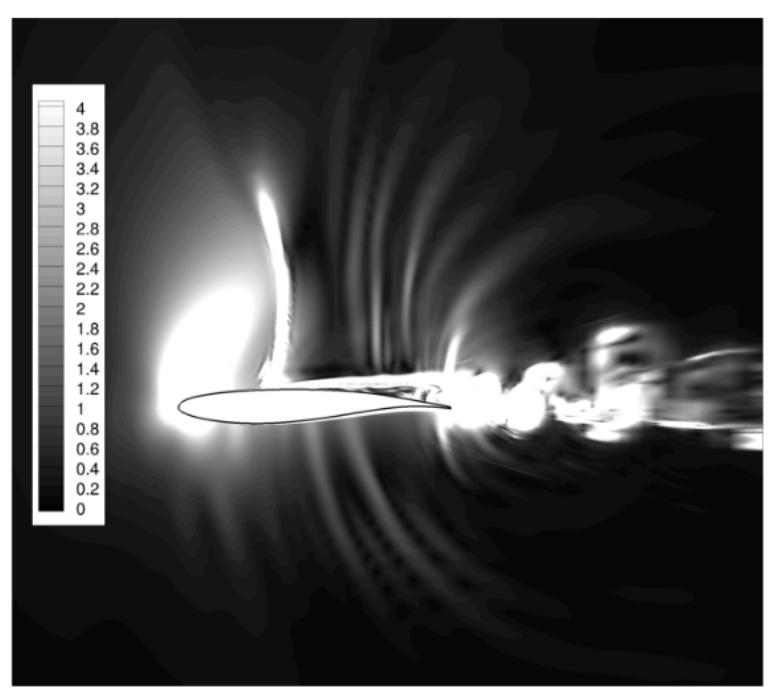

(b) Contours of the norm of the density gradient normalized by the density in a transverse plane

Fig. 5 Visualization of the instantaneous flow field for the ZDES buffet case [29, 31]

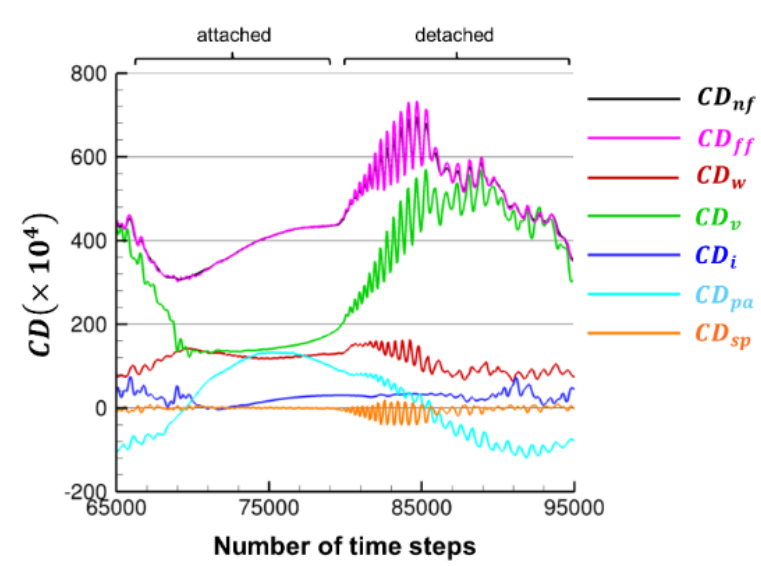

(a) ZDES

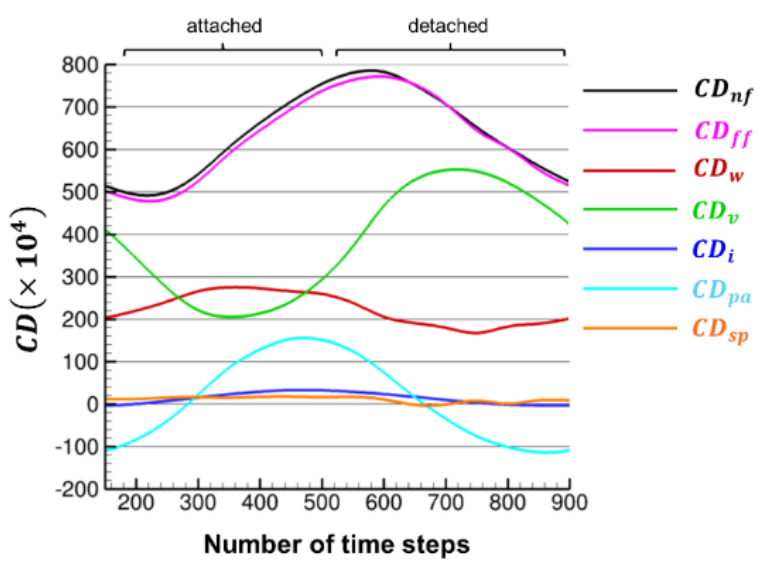

(b) URANS

Fig. 6 Comparison of ZDES and URANS results over one pseudo-period for the buffet case [29, 31]

\section{Applications}

As already discussed in Section 1 . accurate drag computation and decomposition is a major objective of CFD in the aeronautics field. The rapid development of CFD in terms of precision, complexity and numerical techniques has led to the development of a family of far-field drag post-processing codes over the past years at ONERA. The theoretical foundation of the formulations implemented therein has been briefly described in the above sections. From a numerical aspect, the application of these codes includes the post-processing of cell-centered or vertex solutions on structured or unstructured grids, using a variety of different numerical strategies (e.g. the use of overset grids for the treatment of complex geometries). Their domain of application thus covers a wide range of CFD solutions, enabling to account for the full complexity included in computations of realistic geometries. In addition, specific methods are included for the analysis of the airframe and propulsion system contributions to overall aircraft performance. As such, these codes are deeply integrated both in the design cycle of the aeronautical industry and in research-oriented activities within ONERA.

In the research field, far-field drag extraction and decomposition methods have a wide spectrum of application. 


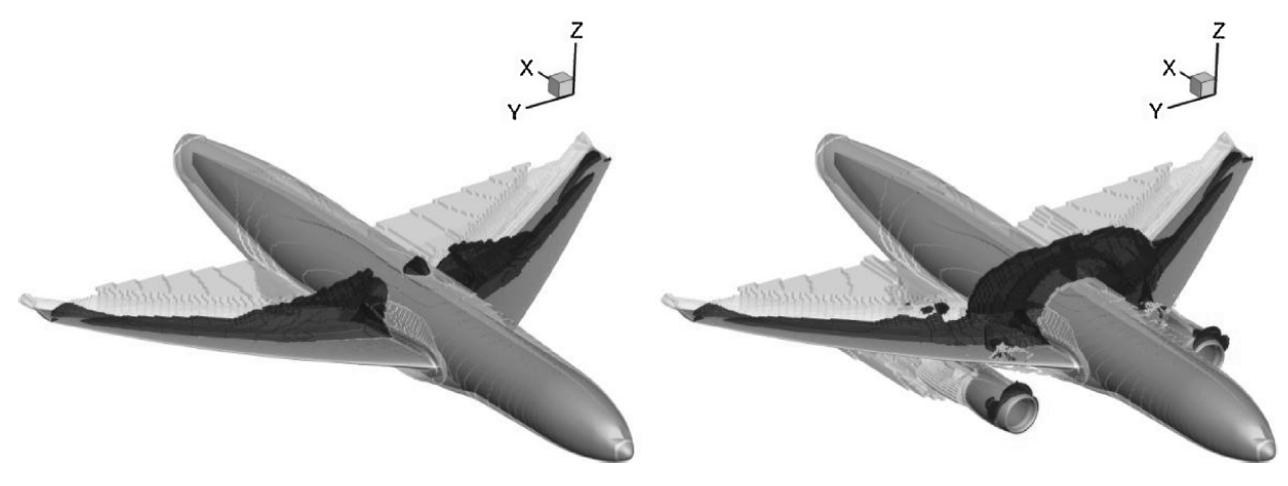

Fig. 7 Overview of integration volumes based on physical criteria for far-field drag extraction for the Common Research Model of the 6th Drag Prediction Workshop [33].

Their more prominent use lies within physical analyses, where the phenomenological decomposition of drag provides a valuable insight [29, 34-36]. Another important aspect is the reduced sensitivity of far-field methods to numerical error, compared to a near-field drag computation. This higher accuracy represents a significant advantage and is one of the principal reasons for their wide application. They are thus commonly employed for the evaluation of the accuracy of numerical methods, whether in terms of classical benchmark cases or in the frame of AIAA Drag Prediction Workshops (Fig. 7] [33, 35, 37]. Furthermore, these codes allow the computation of sensitivity derivatives with respect to far-field drag components. They can thus be coupled with adjoint methods within gradient-based optimization algorithms. Several of their applications concern this aspect, both in purely aerodynamic and aerostructural sensitivity analysis [38-41]. Finally, aerodynamic performance evaluation using these methods is naturally most useful within an aerodynamic design process, in the development of both standard and more innovative configurations [42, 43].

\section{B. Overview of actual studies}

The theoretical formulation based on the van der Vooren formulation made it possible to develop a family of codes today widely used in the European aeronautical industry. The basic formulation was developed initially for subsonic and transonic flows in free-stream conditions. Following the evolution of studies and research carried out in the aeronautical context, this formulation has been extended to more complex flows. However, the research carried out so far has also identified the drawbacks of this far-field approach and has led ONERA to initiate new lines of research. The first drawback is theoretical and is related to the definition of induced drag. The thermodynamic formulation used clearly defines the irreversible drag through the variations of stagnation enthalpy and the production of entropy. Induced drag, called reversible drag, is defined indirectly since it is simply the remainder of the total drag from which the total irreversible drag is subtracted. Thus, this approach does not account for the detail of the velocity vector field and more particularly the vorticity. In order to solve this problem, a complementary approach is the subject of current work. This is related to a formulation based on the velocity vector field, and more particularly the Lamb vector. The second drawback, of practical nature, concerns drag/thrust bookkeeping and is common to all drag extraction methods. Future aircraft configurations very often feature advanced engine integration approaches which are more complex than those encountered on conventional configurations of current aircraft. These configurations often lead to a strong interaction between the airframe and the propulsion system, and may not always clearly distinguish the engine part from the rest of the aircraft (e.g. boundary-layer ingestion). This has motivated the investigation of an exergy-based approach, which is currently the subject of theoretical work. Although the concept of exergy corresponds to a new paradigm, it allows to some extent to evaluate drag components. These two lines of research are presented in the following two paragraphs.

\section{Lamb vector formulation}

The Lamb vector formulation (vortex-force theory) belongs to the vorticity-based formulations, which form another family of far-field methods. In the very recent years, it has been subject to intense research [44-47] since the theory is based on an exact formula which provides the three components of the far-field drag along with the lift in compressible viscous flows. It follows Prandtl's developments on the vortex force [48] and his inviscid lifting-line theory. The exact 
expression of the aerodynamic force is [47]:

$$
\mathbf{F}=\mathbf{F}_{\rho l}+\mathbf{F}_{m_{\rho}}+\mathbf{F}_{S_{e}}+\mathbf{F}_{\tau}
$$

with

$$
\begin{aligned}
\mathbf{F}_{\rho l} & =-\int_{\Omega} \rho \mathbf{l} d v \\
\mathbf{F}_{m_{\rho}} & =-\frac{1}{\mathcal{N}-1} \int_{\Omega} \mathbf{r} \times\left(\nabla \rho \times \nabla\left(\frac{q^{2}}{2}\right)\right) d v \\
\mathbf{F}_{S e} & =-\frac{1}{\mathcal{N}-1} \int_{S_{e}} \mathbf{r} \times(\mathbf{n} \times \rho \mathbf{l}) d S \\
\mathbf{F}_{\tau} & =\frac{1}{\mathcal{N}-1} \int_{S_{e}} \mathbf{r} \times(\mathbf{n} \times \nabla . \tau) d S+\int_{S_{e}} \tau . \mathbf{n} d S
\end{aligned}
$$

where $\mathcal{N}=2,3$ is the physical space dimension and $\mathbf{r}$ is the position vector. For a large enough control volume, $S_{e}$ retreats to flow regions where viscous stresses become negligible and $\mathbf{F}_{\tau}$ can be neglected [45, 49]. Then, the decomposition proposed by Mele and Tognaccini [47] is the following:

$$
\begin{aligned}
L^{\text {Mele }} & =\mathbf{k} \cdot\left(\mathbf{F}_{\rho l}+\mathbf{F}_{m_{\rho}}\right) \\
D_{i}^{\text {Mele }} & =\mathbf{i} \cdot\left(\mathbf{F}_{\rho l}+\mathbf{F}_{m_{\rho}}\right) \\
D_{P}^{\text {Mele }} & =\mathbf{i} \cdot \mathbf{F}_{S_{e}}
\end{aligned}
$$

However, the presence of the position vector raises the following question: is the force decomposition dependent on the reference point adopted for the computation of moments? Indeed, the shift of $\mathbf{r}$ by a constant $\mathbf{r}_{0}$ (i.e. $\mathbf{r} \rightarrow \mathbf{r}+\mathbf{r}_{0}$ ) generates nonzero additional terms in $\mathbf{F}_{m_{\rho}}$ and $\mathbf{F}_{S e}$ :

$$
\begin{aligned}
\mathbf{F}_{S e} & =-\frac{1}{\mathcal{N}-1} \int_{S_{e}} \mathbf{r} \times(\mathbf{n} \times \rho \mathbf{l}) d S-\frac{\mathbf{r}_{0}}{\mathcal{N}-1} \times \int_{S_{e}} \mathbf{n} \times \rho \mathbf{l} d S \\
\mathbf{F}_{m_{\rho}} & =-\frac{1}{\mathcal{N}-1} \int_{\Omega} \mathbf{r} \times\left(\nabla \rho \times \nabla\left(\frac{q^{2}}{2}\right)\right) d v+\frac{\mathbf{r}_{0}}{\mathcal{N}-1} \times \int_{S_{e}} \mathbf{n} \times \frac{q^{2}}{2} \nabla \rho d S
\end{aligned}
$$

Therefore, an objective force decomposition remains impossible as long as the contribution of $\mathbf{F}_{m_{\rho}}$ and $\mathbf{F}_{S e}$ to the total force varies with the location of the reference point. A necessary condition for invariance is:

$$
\int_{S_{e}} \mathbf{n} \times \rho \mathbf{l} d S=\int_{S_{e}} \mathbf{n} \times \frac{q^{2}}{2} \nabla \rho d S=\mathbf{0}
$$

Fournis et al. [50, 51] showed that, due to the far-field symmetry properties satisfied by the flow field, the condition (41) is progressively verified when the size control volume is increased and when $S_{e}$ is sufficiently far from the body surface. The study of the symmetries was split into two parts: the symmetries in the wake based on the presence of the counter-rotating trailing vortices, and the symmetries up and downstream the aircraft fulfilled by the quantity $\frac{q^{2}}{2} \nabla \rho$. The aim was then to find a way to enforce those symmetries everywhere in the flow field such that the force decomposition becomes independent of the reference point even when $S_{e}$ is closer to the body surface. By doing so, the dependence on the reference point completely disappears. The new reference-point-invariant formulation was then applied to transonic flows.

Later on, the relation between the vortex-force theory, the Kutta-Joukowski (KJ) lift theorem and Maskell's lift-induced drag formula was emphasized in compressible flows [52]. Since then, a revised decomposition valid in transonic flows has been developed at ONERA in collaboration with the University of Naples. This formulation is presented in detail in another paper of the current conference [53]:

$$
\begin{aligned}
& L^{\text {ONERA }}=\mathbf{k} \cdot\left(\mathbf{F}_{\rho l}+\mathbf{F}_{m_{\rho}}-\mathbf{F}_{\nabla \rho}\right) \\
& D_{i}^{\text {ONERA }}=\mathbf{i} \cdot\left(\mathbf{F}_{\rho l}+\mathbf{F}_{m_{\rho}}-\mathbf{F}_{\nabla \rho}\right) \\
& D_{P}^{\text {ONERA }}=\mathbf{i} \cdot\left(\mathbf{F}_{S_{e}}+\mathbf{F}_{\tau}+\mathbf{F}_{\nabla \rho}\right)
\end{aligned}
$$


with

$$
\mathbf{F}_{\nabla \rho}=\frac{1}{\mathcal{N}-1} \int_{S_{e}} \mathbf{r} \times\left(\mathbf{n} \times \frac{q^{2}-u_{\infty}^{2}}{2} \nabla \rho\right) d S
$$

This new formulation bridges the gap between the vortex-force theory and the pioneering formulations: the KuttaJoukowski theorem, Maskell's formula and Betz's formula. In fact, it was noticed that these classical analyses are encompassed for the first time in a single expression:

$$
\mathbf{F}=\mathbf{F}_{\rho l}+\mathbf{F}_{m_{\rho}}-\mathbf{F}_{\nabla \rho}+\mathbf{F}_{S_{e}}+\mathbf{F}_{\tau}+\mathbf{F}_{\nabla \rho}
$$

with

$$
\begin{aligned}
\mathbf{F}_{\rho l}+\mathbf{F}_{m_{\rho}}-\mathbf{F}_{\nabla \rho} & =\overbrace{-\rho_{\infty} u_{\infty}\left(\Gamma^{*}+\delta \Gamma^{*}\right) \mathbf{k}}^{\text {Compressible KJ formula }}+\underbrace{\frac{1}{2} \int_{W} \rho\left(v^{2}+w^{2}-\delta u^{2}\right) d S}_{\text {Compressible Maskell formula }} \mathbf{i} \\
\mathbf{F}_{S_{e}}+\mathbf{F}_{\tau}+\mathbf{F}_{\nabla \rho} & =\int_{S_{e}} \tau \cdot \mathbf{n} d S+\underbrace{\int_{S_{e}}\left(p_{t_{\infty}}-p_{t}\right) \mathbf{n} d S+\frac{u_{\infty}^{2}}{2} \int_{S_{e}}\left(\rho-\rho_{\infty}\right) \mathbf{n} d S}_{\text {Compressible Betz formula }}
\end{aligned}
$$

Here, the velocity vector is split into $\mathbf{q}=u_{\infty} \mathbf{i}+\delta \mathbf{q}$ with $\delta \mathbf{q}=\left(\begin{array}{lll}\delta u & v & w\end{array}\right)^{T}, W$ is a wake plane on which $\mathbf{n}=\mathbf{i}, \Gamma^{*}$ is a circulation and $\delta \Gamma^{*}$ is a viscous lift penalty [54]:

$$
\Gamma^{*}=-\mathbf{j} . \int_{S_{e}} \mathbf{n} \times \frac{\rho}{\rho_{\infty}} \delta \mathbf{q} d S \quad \text { and } \quad \delta \Gamma^{*}=\frac{1}{u_{\infty}} \int_{W} \frac{\rho}{\rho_{\infty}} w \delta u d S
$$

The new formulation also provides practical advantages since the force decomposition is independent of the reference point chosen for the calculation of moment transformations. Moreover, it improves the drag decomposition on aircraft configurations, and the computation can be performed in a better-refined part of the grid. The evolution of the drag decomposition with respect to the size of the control volume is given in Fig. 8 on the Common Research Model in cruise flight conditions. In Fig. 88, the size of the control volume is increased by increasing $d$, the distance between $S_{e}$ and $S_{A}$. Besides, $c_{r e f}$ is the mean aerodynamic chord, VdV refers to van der Vooren's approach, MSK to the compressible Maskell formula and BETZ to the compressible Betz formula.

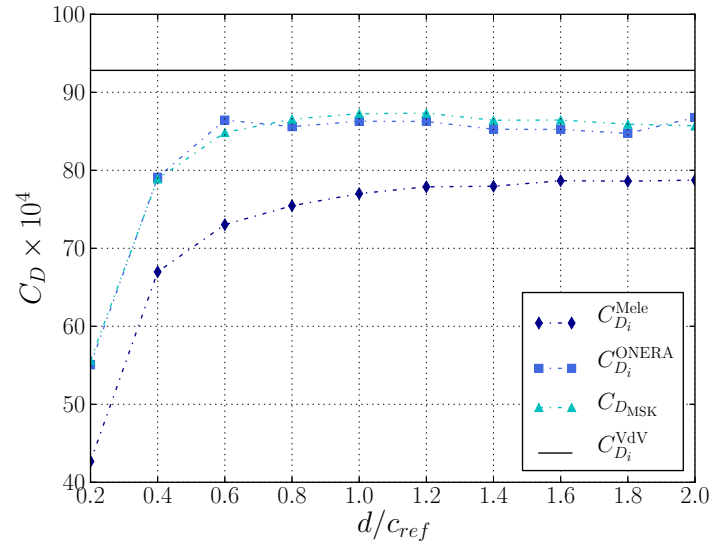

(a) Lift-induced drag

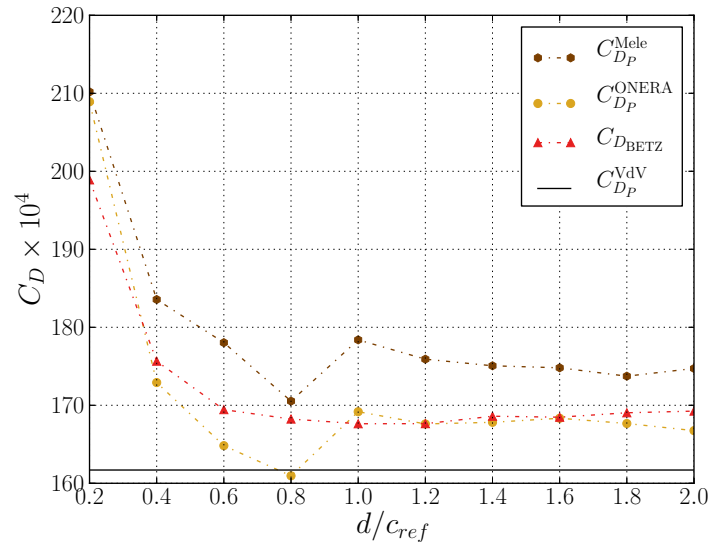

(b) Profile drag

Fig. 8 Lift-induced and profile drag predictions on the Common Research Model using the Lamb vector formulations, $M_{\infty}=0.85, R e=5 \times 10^{6}, C_{L} \approx 0.5[53]$ 


\section{Exergy formulation}

Due to stringent requirements, imposed by the need to reach increased aerodynamic efficiency or to meet regulation constraints, aircraft have evolved towards extremely complex systems that require adapted methodologies and tools for an efficient design process. Indeed, concepts involving a highly integrated propulsive system into the airframe (e.g. boundary-layer ingestion) may make it difficult to perform a clear distinction between the thrust and drag components of the total streamwise force. A more appropriate performance evaluation of these configurations requires a new paradigm. Drela developed the power balance method based on mechanical power and the flow of kinetic energy, which is appropriate for the analysis of such configurations [55]. Another theoretical formulation based on an exergy balance has then been developed at ONERA for assessing the aero-thermo-propulsive performance of future aircraft configurations [56]. As opposed to the separation of energy into mechanical and thermal (partially convertible to work), the notion of exergy is used to refer to the maximum theoretically recoverable work which can be extracted from the system by returning it to the reference conditions.

It consists in the combination of a momentum balance and a fluid flow analysis involving the first and second laws of thermodynamics, resulting in an exergy balance within a control volume. This balance can be expressed in the general form:

$$
\dot{\mathcal{E}}_{\text {prop }}+\dot{\mathcal{E}}_{q}=W \dot{\Gamma}+\dot{\mathcal{E}}_{m}+\dot{\mathcal{E}}_{t h}+\dot{\mathcal{A}}_{t o t}
$$

The left-hand side of the balance represents the inflow of exergy in the control volume, by the propulsive system or non-adiabatic boundaries. Its right-hand side terms represent the power consumption due to an off-equilibrium force balance, the outflow of mechanical and thermal exergy from the control volume and finally the destruction of exergy within the control volume due to irreversible phenomena (viscous effects, thermal mixing, shockwaves).

Multiple studies have been conducted to evaluate this new exergy balance formulation on a wide range of configurations [56-59]. More recent investigations have also focused on the understanding and quantification of the influence of numerical effects, which is essential for the application of the method to the analysis of numerical solutions in an industrial scale [60, 61].

The restriction of the formulation to unpowered configurations yields connections to some well-known far-field drag expressions and shows that their underlying theory can be related to exergy considerations. Studies of unpowered configurations have thus allowed the detailed investigation of viscous and shock wave phenomena from 2D airfoils, as well as induced drag from a wing-body aircraft geometry [56, 59]. This basis has also been used by other research groups for the evaluation of an exergy-based drag coefficient, with an interest in its application to the analysis of wind-tunnel experiments [62].

The exergy balance formulation has also been applied to several powered configurations, with wake or boundary layer ingestion (BLI). Two more recent examples of application are briefly presented below.

Propulsive fuselage The FFX (far-field exergy) tool was applied to an axisymmetric academic propulsive fuselage shape in order to assess its aero-propulsive performance [63]. Three different configurations were investigated (see Fig. 97:

- An isolated nacelle and fuselage (equivalent to a nacelle positioned infinitely away from the fuselage)

- The fuselage with the nacelle positioned downstream (far enough not to disturb the rear fuselage pressure field), in a more academic wake ingestion configuration

- The installed configuration (same wetted area between configurations 1 and 2)

Computations were carried out with the elsA solver [26], using a body-force method (Hall-Thollet model) to simulate the fan. Computations were performed for various fan RPM, as illustrated in Fig. 9. $R_{x}$ corresponds to the streamwise net force coefficient including the forces acting the wetted surface and the fan traction. $R_{x}=0$ means that the fan thrust balances the drag of the body, while $R_{x}<0$ means an excess of thrust.

Results of the CFD computations and exergy post-processing with the FFX code on the three configurations are presented in Fig. 9 The terms $\dot{\mathcal{E}}_{m}$ and $\dot{\mathcal{E}}_{t h}$ represent the exergy that could be recovered in a reversible manner, while $\dot{\mathcal{A}}_{t o t}$ corresponds to the destruction of exergy in an irreversible process. $W \dot{\Gamma}$ values are very similar between configurations as comparisons are performed at the same streamwise net force coefficient $R_{x}$. Mechanical exergy outflow is decomposed as $\dot{\mathcal{E}}_{m}=\dot{E}_{u}+\dot{E}_{v w}+\dot{E}_{p}$, its first two components concerning respectively streamwise and transversal kinetic energy outflow and its third component being the boundary pressure-work rate. For $R_{x}=0$ with BLI, the fan fills the momentum deficit of the fuselage. In this case, there is no excess or deficit of axial velocity, so the velocity downstream of the fan corresponds to a Mach number very close to the free-stream value (Fig. 9 bottom field visualization), so $\dot{E}_{u} \simeq 0$. On the other hand, it is also $\dot{E}_{v w}=0$ (axi-symmetric configuration) and $\dot{E}_{p} \simeq 0$ far enough 

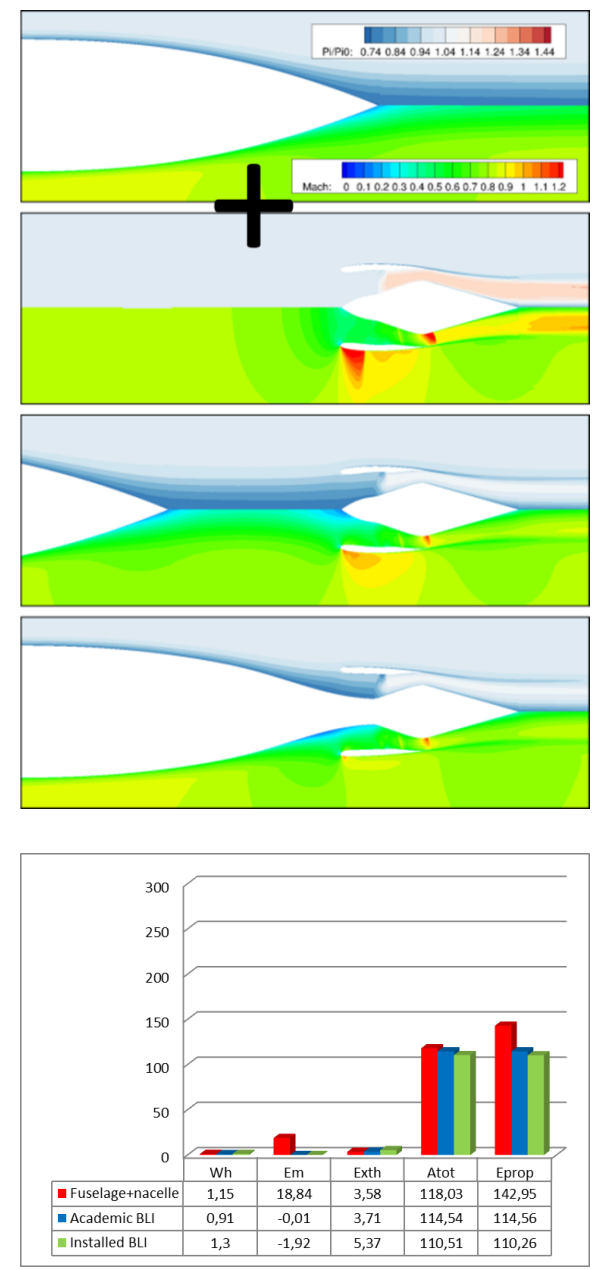
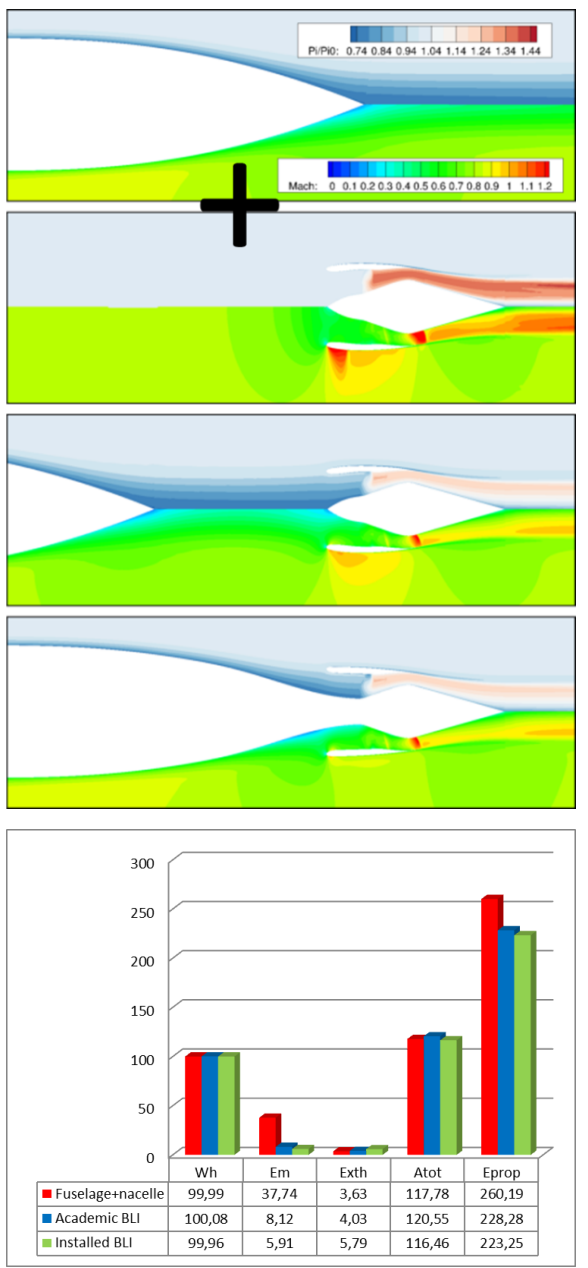

Fig. 9 Flow field visualizations corresponding to the different configurations at $M_{\infty}=0.82$ and the corresponding exergy balance at $R_{x}=0$ (left) or $R_{x}=-100$ d.c. (right). Fan stage modeled via a body-force source term method and exergy terms expressed in the form of non-dimensional coefficients, in power counts $\left(\times 10^{-4}\right)[63]$.

from the configuration, so $\dot{\mathcal{E}}_{m} \simeq 0$. On the contrary, the fuselage + nacelle configuration is wasting $\dot{\mathcal{E}}_{m} \simeq 18$ p.c. of exergy, which could have been recovered by BLI. As a consequence, that configuration requires a higher propulsive exergy inflow $\dot{\mathcal{E}}_{\text {prop }}$ than the others. The same trend appears for $R_{x}=-100$, which corresponds to a more realistic aircraft propulsive ratio (this corresponds approximately to the thrust needed for a wing+fuselage configuration). In this case the benefit of BLI on $\dot{\mathcal{E}}_{m}$ is even larger, whereas the impact on $\dot{\mathcal{A}}_{t o t}$ remains small. Moreover, in the case of the fuselage+nacelle configuration, viscous wakes are dissipated separately in contrast an installed BLI case, which increases total anergy $\dot{\mathcal{A}}_{t o t}$ in particular via its viscous component (Fig. 9). In summary, the wake ingestion and BLI configurations mostly save exergy by reducing the excess of axial velocity of the engine jet, thus reducing the wasted mechanical exergy $\dot{\mathcal{E}}_{m}$. This can be combined with an additional small saving on the dissipated anergy, both leading to an overall reduction of the required propulsive exergy $\dot{\mathcal{E}}_{\text {prop }}$. Such an analysis would not have been possible in a conventional thrust/drag bookkeeping approach, thus illustrating the benefit of the exergy balance (or power balance [55]) for such coupled aero-propulsive configurations.

Aero-thermal performance of a heat sink Another benefit of the exergy balance is its capacity to assess the aero-thermal performance of heat exchangers. That new field of investigation for CFD results has not been fully covered yet. CFD computations are currently performed without coupling with a thermal solver, but such investigations are planned in the future. The configuration investigated here is a straight heat sink (Fig. 10) that can be found on computer CPUs (ventirad), in the secondary stream of aircraft engines (Surface Air-Cooled Oil Cooler). 


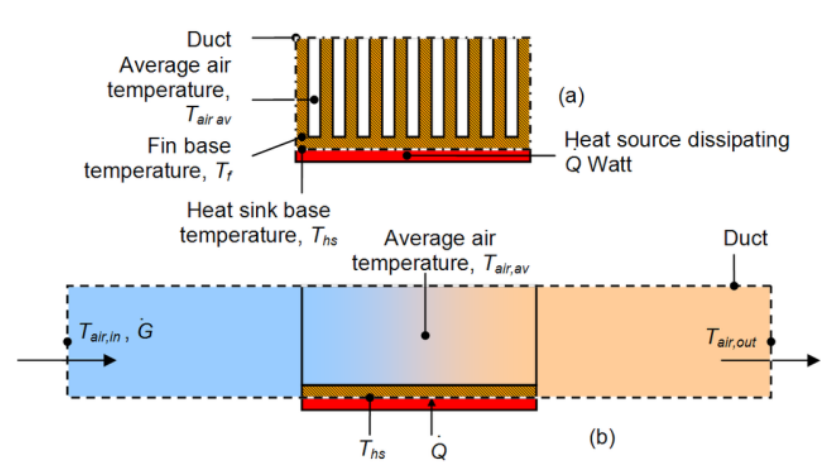

Fig. 10 Schematic illustration of heat sink principle, source: Wikipedia*

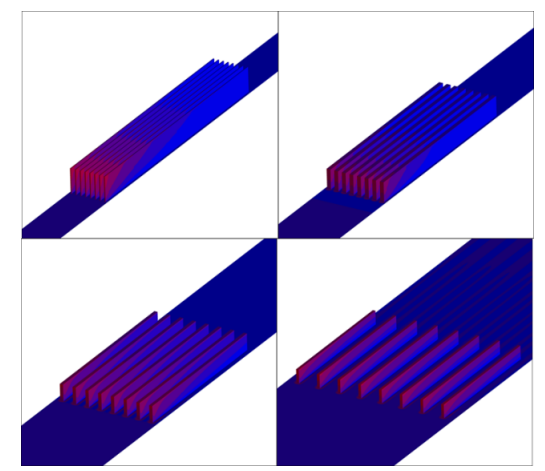

Fig. 11 View of different heat sink configurations with varying fin spacing

\begin{tabular}{|c|c|c|c|c|c|c|c|c|c|}
\hline & Step & Heated Area & Q & Heat flux per fin & Fin number & $W \dot{\Gamma}$ & $\dot{\mathcal{E}}_{m}$ & $\dot{E}_{p}$ & $\dot{\mathcal{E}}_{t h}$ \\
\hline CONF23 & $5 \mathrm{~mm}$ & $13800 \mathrm{~mm}^{2}$ & $3623 \mathrm{~W} / \mathrm{m}^{2}$ & $50 \mathrm{~W}$ & 200 & -168.9 & 5.2 & -26.0 & 27.6 \\
\hline CONF24 & $7.5 \mathrm{~mm}$ & $14425 \mathrm{~mm}^{2}$ & $5199 \mathrm{~W} / \mathrm{m}^{2}$ & $75 \mathrm{~W}$ & 133 & -154.2 & 7.6 & -17.2 & 30.0 \\
\hline CONF25 & $10 \mathrm{~mm}$ & $15050 \mathrm{~mm}^{2}$ & $6645 \mathrm{~W} / \mathrm{m}^{2}$ & $100 \mathrm{~W}$ & 100 & -143.1 & 7.6 & -12.9 & 30.2 \\
\hline CONF26 & $15 \mathrm{~mm}$ & $16300 \mathrm{~mm}^{2}$ & $9202 \mathrm{~W} / \mathrm{m}^{2}$ & $150 \mathrm{~W}$ & 67 & -128.1 & 7.1 & -8.6 & 29.0 \\
\hline CONF27 & $20 \mathrm{~mm}$ & $17550 \mathrm{~mm}^{2}$ & $11396 \mathrm{~W} / \mathrm{m}^{2}$ & $200 \mathrm{~W}$ & 50 & -122.2 & 6.7 & -6.4 & 28.0 \\
\hline CONF28 & $25 \mathrm{~mm}$ & $18800 \mathrm{~mm}^{2}$ & $13298 \mathrm{~W} / \mathrm{m}^{2}$ & $250 \mathrm{~W}$ & 40 & -116.8 & 6.6 & -5.1 & 27.4 \\
\hline CONF29 & $30 \mathrm{~mm}$ & $20050 \mathrm{~mm}^{2}$ & $14963 \mathrm{~W} / \mathrm{m}^{2}$ & $300 \mathrm{~W}$ & 33 & -114.7 & 6.5 & -4.2 & 27.2 \\
\hline CONF30 & $40 \mathrm{~mm}$ & $22550 \mathrm{~mm}^{2}$ & $17738 \mathrm{~W} / \mathrm{m}^{2}$ & $400 \mathrm{~W}$ & 25 & -111.3 & 6.5 & -3.1 & 27.6 \\
\hline
\end{tabular}

Table 3 Fin geometry, heat transfer parameters, and exergy balance coefficients for $x T P=4 \mathrm{~m}$.

Once again an academic configuration is preferred to a more complex test case at this stage. The configuration is a rectangular fin with an imposed heat flux over its surface. A simple parametric study was performed for a given fin geometry and different fin spacings (Fig. 11). The fin dimensions are $L=250 \mathrm{~mm}, W=1 \mathrm{~mm}$ and $H=25 \mathrm{~mm}$. Consequently, when considering a heat sink geometry of a total width of $1 \mathrm{~m}$, the number of fins increases or decreases, and so a different area is available for the dissipation of the heat flux. The heat flux is thus imposed such that a total of $10 \mathrm{~kW}$ is dissipated on a width of $1 \mathrm{~m}$ of heat sink (Table 3). All computations are performed with elsA solver, using a $3 \mathrm{~m}$ viscous flat plate upstream of the fin, in order to allow the development of a boundary layer with a thickness of approximately $30 \mathrm{~mm}$. The Mach number is set to 0.3 , and the Spalart-Allmaras turbulence model is used. The computation is run for a number of iterations larger than usual, in order to eliminate the eventual influence of errors due to insufficient convergence in the resulting exergy balance.

After analyzing the exergy balance for different positions of the downstream limit of the control volume $(x T P)$, it was chosen to perform the analysis at $x T P=4 \mathrm{~m}$, a value beyond which $\dot{E}_{p}$ remains relatively constant (Fig. 12 shows an example for two heat sink configurations). The fin is placed between $x T P=3 \mathrm{~m}$ and $x T P=3.25 \mathrm{~m}$, and is the only part of the wall surface on which a heat flux is imposed. Fig. 13 plots the values of Table 3 All coefficients are relative to a one-meter-wide heat sink, so that they can be directly compared. First, note that a maximum is obtained for $\dot{\mathcal{E}}_{m}+\dot{\mathcal{E}}_{t h}$ (sum of mechanical and thermal exergy outflow). This means the configurations with a fin spacing of $7.5 \mathrm{~mm}$ and $10 \mathrm{~mm}$ waste more exergy. Results of this exergy analysis indicate that to minimize the outflow of exergy fin spacing should be either decreased (less than $5 \mathrm{~mm}$ ), or further increased (>30mm). However, current computations do not include any thermal coupling, and a significant decrease of fin spacing could lead to large local temperatures. The purpose of the present analysis is just to show how to handle the FFX tool in such a case, despite the fact that current CFD results are not suitable to drive a general conclusion on optimal fin spacing. The maximum of $\dot{\mathcal{E}}_{m}+\dot{\mathcal{E}}_{t h}$ corresponds to a maximum for both $\dot{\mathcal{E}}_{m}$ and $\dot{\mathcal{E}}_{t h}$. There is no local maximum of $\dot{E}_{u}$, because $\dot{E}_{u}$ corresponds to the wake of the fin. An increased number of fins leads to a larger wake defect. $\dot{E}_{p}$ corresponds to the pressure interaction between fins, and shows an opposite behavior with respect to $\dot{E}_{u}$. When coupled with a thermal code, such an analysis can enable to find optimum spacings, fin aspect ratio, heat flux values for such common heat sink designs. A next step would be to optimize the shape of the fin itself, using airfoils instead of rectangular shapes. FFX will thus provide a new perspective which will be precious in the design and analysis of the aero-thermal performance of air systems.

"https://en.wikipedia.org/wiki/Heat_sink 

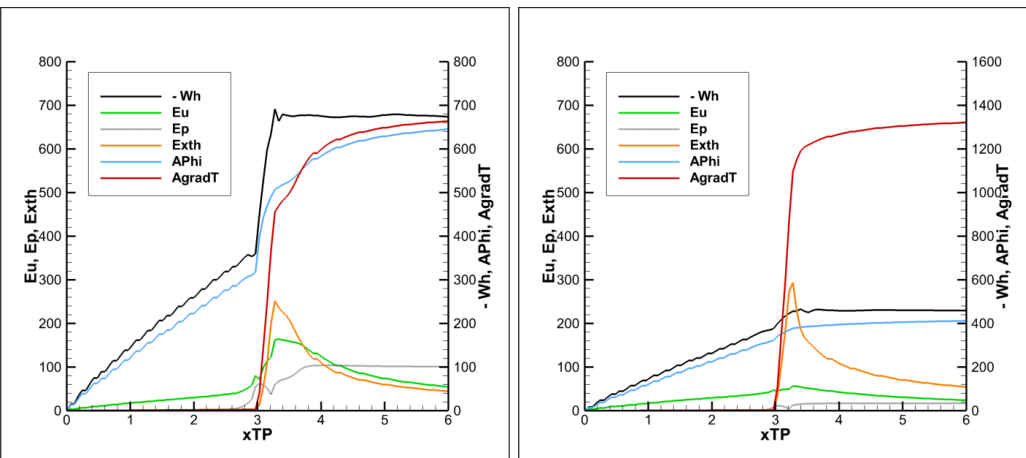

Fig. 12 Downstream variation of the exergy balance for the CONF23 (left) and CONF29 (right) geometries

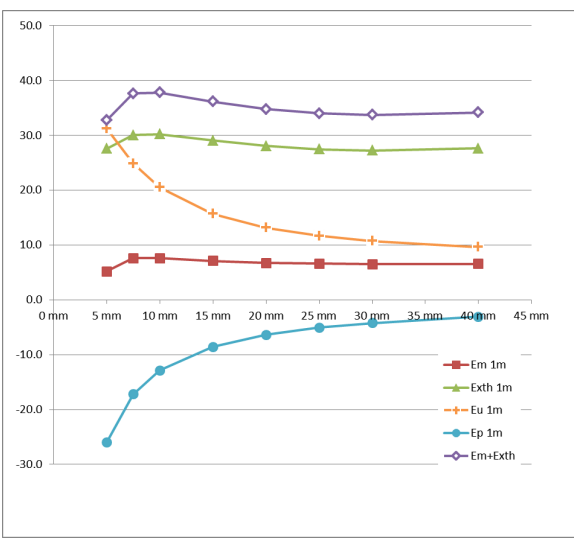

Fig. 13 Exergy balance for varying fin spacing at $x T P=4 \mathbf{m}$

Current work focuses on the extension of the formulation to more complex flows (e.g. rotating frame of reference), in order to be able to apply it to more realistic aero-propulsive configurations. In parallel, the study of certain terms of the exergy balance is being carried out in order to better assess their link with classical mechanical theories. In particular, the term of wave anergy, related to the presence of shock waves in the flow, is not directly equivalent to wave drag and a rigorous link between the two terms requires further investigation. This should then allow to extend the approach to supersonic flows.

\section{Experimental}

Wind tunnel tests remain important milestones during design phases to validate the performance increments of novel aircraft configurations, even though numerical methods have made important progress over the past decades. Thus, far-field drag decomposition methods have also been developed to be applied during wind tunnel test campaigns. The direct measurement of total mean drag acting on a model using a balance has been tried and tested, and is nowadays customarily found on industrial-scale experimental platforms. This method however only gives a global account of drag, and makes it impossible to investigate its various sources.

\section{A. Five-hole probe wake measurements}

Within this context, several drag decomposition formulations have been proposed (cf. Ref. [64] for a review and cross comparison of their performance and domains of applicability). Based on a global balance of momentum, these formulations allow for a finer description of drag, from the knowledge of the complete velocity field and stagnation pressure in the wake of the model. Such data is typically acquired with five-hole probes. The formulations developed for wind tunnels are different from those in the numerical field. The objective of these formulas is to provide accurate drag values from the smallest possible measurement volume. Today, thanks to our formulations, measurements are concentrated in the viscous wake and vorticity in a downstream plane to the model.

$$
\begin{gathered}
C_{D}^{\text {Profile }}=\frac{1}{S_{\text {ref }}} \int_{S_{w}}\left(-\frac{2}{\gamma M_{\infty}^{2}} \frac{\delta p_{i}}{p_{i_{\infty}}}+\left(M_{\infty}^{2}-1\right) \frac{\delta u^{2}}{u_{\infty}^{2}}\right) d S \\
C_{D}^{\text {Induced }}=\frac{1}{S_{\text {ref }}} \int_{S_{e}} \frac{v^{2}+w^{2}}{u_{\infty}^{2}} d S=\frac{1}{S_{\text {ref }}} \int_{S_{w}} \frac{\psi \omega}{u_{\infty}^{2}} d S
\end{gathered}
$$

Formulations (wave drag extraction from the profile drag) and experimental methodologies have been improved to give accurate results. In particular, the extraction of the wave drag from the profile drag is obtained by an original method based on POD.

Numerous tests were carried out on different geometric configurations and different experimental conditions (model 

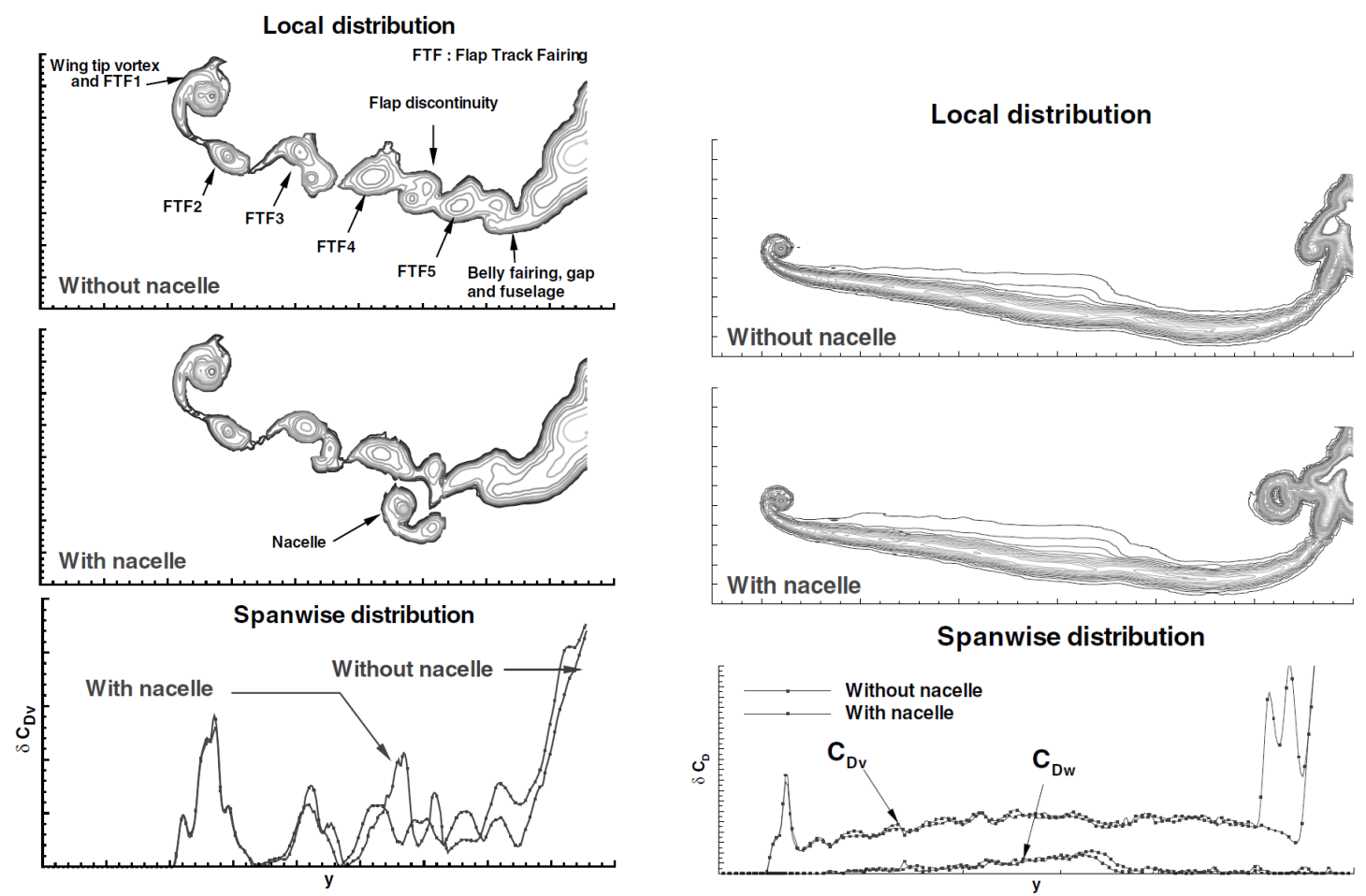

Fig. 14 Typical local and spanwise profile drag distributions obtained using 5-hole probe wake measurements on a high-lift aircraft configuration in subsonic flow (left) and an airframe-nacelle configuration in transonic flow (right) [64].

scale and Mach number) in ONERA and research/industrial partner wind tunnels. Fig. 14]shows typical results obtained using five-hole probe wake measurements.

Five-hole probes are however intrusive and extremely expensive to operate, which may explain why they have been seldom used at an industrial level. Furthermore, owing to their slow response time, five-hole probes forbid instantaneous load measurements, which is necessary when the model is subject to quick unsteady phenomena such as vortex shedding. In contrast, the continuous improvements made to PIV over the past decades have made it the de facto standard when performing measurements in fluids.

\section{B. Stereo-PIV wake measurements}

As for five-hole measurements, the cornerstone of the method consists in performing a global balance of momentum over a control volume $\Omega$. Considering a steady mean flow and time averaged quantities, the mean aerodynamic load $\mathbf{F}$ acting on the model is found to be:

$$
\mathbf{F}=-\int_{\partial \Omega} \rho \mathbf{q q} \cdot \mathbf{n} d S-\int_{\partial \Omega} \rho<\mathbf{q}^{\prime} \otimes \mathbf{q}^{\prime}>. \mathbf{n} d S-\int_{\partial \Omega} p \mathbf{n} d S-\int_{\partial \Omega} \mu\left[\nabla \mathbf{q}+(\nabla \mathbf{q})^{T}\right] . \mathbf{n} d S
$$

where $\mathbf{q}$ and $p$ respectively represent the mean velocity and mean static pressure fields, $\rho$ denotes density and $\mu$ denotes dynamic viscosity. The term $\left\langle\mathbf{q}^{\prime} \otimes \mathbf{q}^{\prime}>\right.$ refers to the Reynolds stress tensor, with $\mathbf{q}^{\prime}$ being the turbulent velocity fluctuation. The effects of viscous friction along $\partial \Omega$ are considerably smaller than any other term (about two orders of magnitude here), hence they can be safely dropped. Assuming that the lateral boundaries are sufficiently far from the model, there is no flux of momentum across them, and the global balance simplifies to evaluating the difference of momentum between the up- and downstream planes of the control volume. This assumption also implies that the turbulent stresses will be non-negligible only in the downstream plane. Finally, the downstream plane is assumed to be located sufficiently far away from the model, such that the quantities measured downstream differ only slightly from those upstream. Within this small perturbation framework, one can define $p_{i}=p_{i_{\infty}}+\delta p_{i}\left(p_{i_{\infty}}\right.$ being the freestream stagnation pressure) and $\mathbf{q}=\left(u_{\infty}+\delta u\right) \mathbf{i}+v \mathbf{j}+w \mathbf{k}$, where the perturbations $\delta p_{i} / p_{i_{\infty}}, \delta u / u_{\infty}, v / u_{\infty}$ and $w / u_{\infty}$ are all 

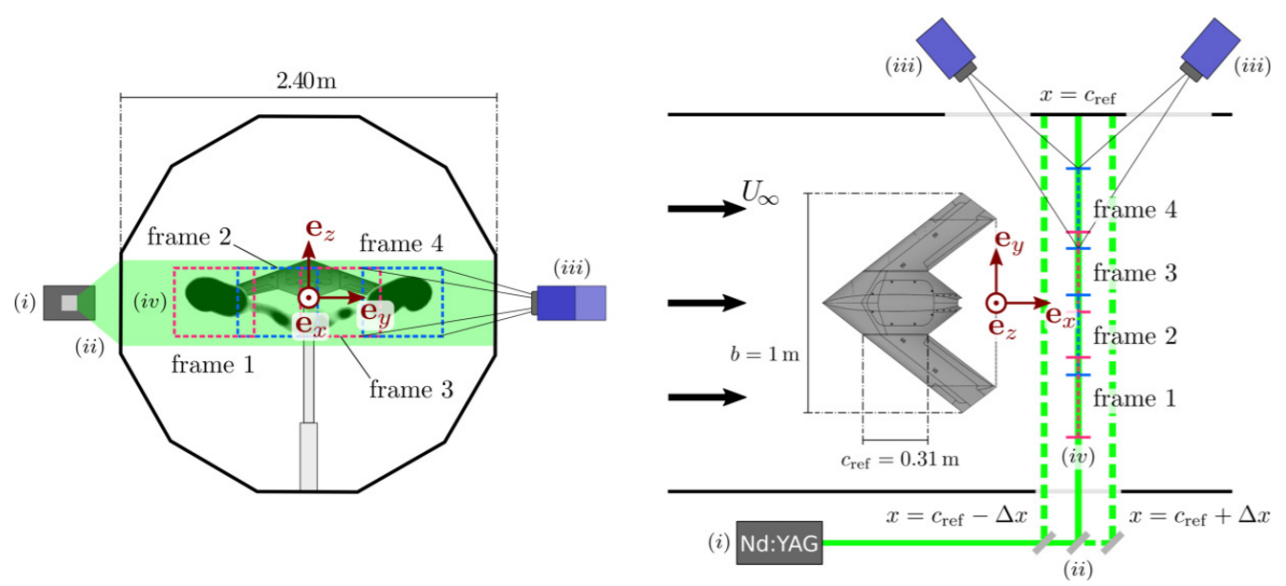

Fig. 15 Overview of the stereo-PIV setup [65].

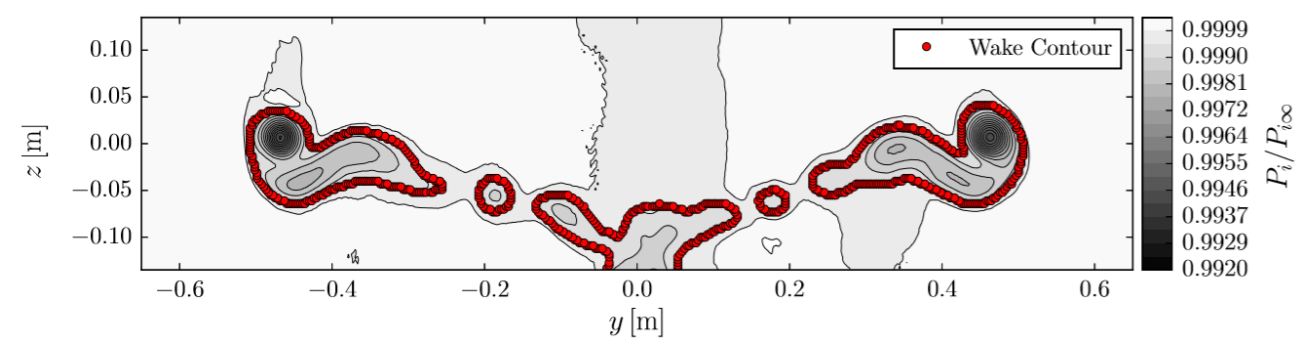

Fig. 16 Wake contour for $\alpha=13^{\circ}[\mathbf{6 5}$.

small compared to one. Owing to a Taylor expansion up to second order, the equation could be re-written in the form:

$$
C_{D}=C_{D}^{\text {Profile }}+C_{D}^{\text {Induced }}+C_{D}^{\text {Turbulent }}
$$

with

$$
\begin{aligned}
C_{D}^{\text {Profile }}= & \frac{1}{S_{\text {ref }}} \int_{S_{w}}-\frac{2}{\gamma M_{\infty}^{2}} \frac{\delta p_{i}}{p_{i_{\infty}}}+\left(M_{\infty}^{2}-1\right) \frac{\delta u^{2}}{u_{\infty}} d S \\
C_{D}^{\text {Induced }}= & \frac{1}{S_{\text {ref }}} \int_{S_{e}} \frac{v^{2}+w^{2}}{u_{\infty}^{2}} d S=\frac{1}{S_{\text {ref }}} \int_{S_{w}} \frac{\psi \omega}{u_{\infty}^{2}} d S \\
& C_{D}^{\text {Turbulent }}=-\frac{2}{S_{\text {ref }}} \int_{S_{w}} \frac{\rho}{\rho_{\infty}} \frac{<u^{\prime} u^{\prime}>}{u_{\infty}^{2}} d S
\end{aligned}
$$

The integration domain of the phenomenological breakdown is reduced to the actual wake $S_{w}$. This particular feature of the phenomenological method makes it quite appealing experimentally speaking, as it drastically reduces the span of the domain to capture. There is however a tradeoff with reducing the integration domain to the wake. Indeed, doing so focuses all the information scattered throughout space in a much smaller region, and especially in the viscous cores of the wake vortices.

The knowledge of the stagnation pressure in the wake plane is required in order to evaluate drag. Computing the pressure field from PIV velocity data under the constraint of an incompressible flow is a long standing issue. Two strategies can be conducted for the static pressure calculation, the first one correspond to solve directly a Poisson equation and the second one, which is adopted here, relies on integrating the pressure gradient of the Navier-Stokes equations [66]:

$$
-\nabla p=\rho \mathbf{q} . \nabla \mathbf{q}+\rho \nabla .\left\langle\mathbf{q}^{\prime} \otimes \mathbf{q}^{\prime}>-\mu \Delta \mathbf{q}\right.
$$

The different terms composing the pressure gradient are calculated from the PIV measurements by spatial second order schemes. The pressure field is then obtained by minimizing a functional built on the difference of the pressure gradient 


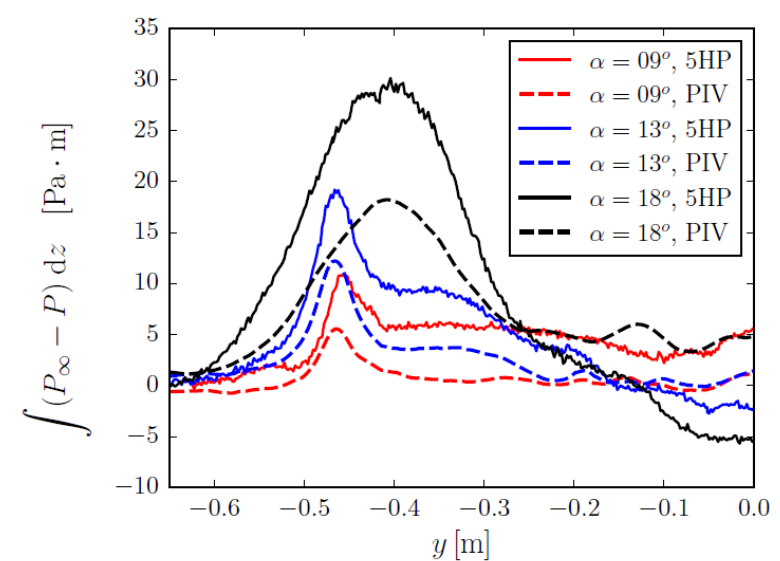

(a) Static pressure

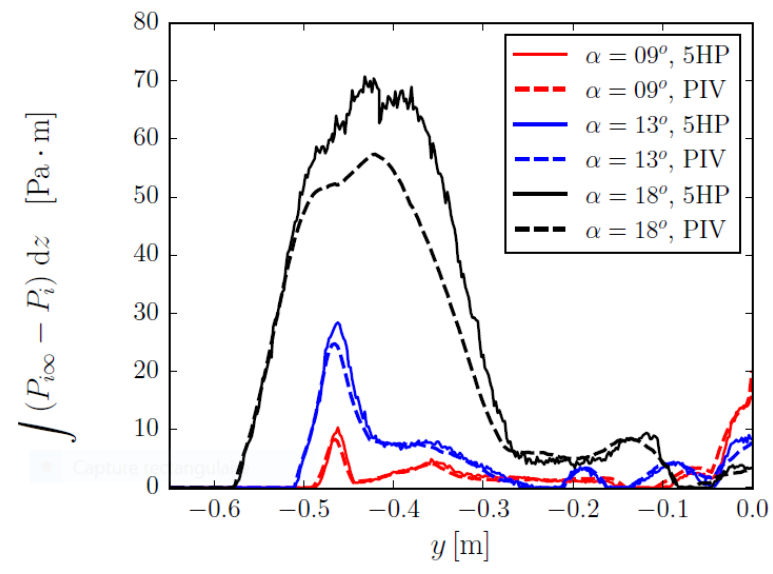

(b) Stagnation pressure

Fig. 17 Spanwise of pressure: Solid and dashed lines refer to 5-hole probe measurements and to reconstruction based and PIV measurements [65].

based on the PIV measurements and the estimated pressure gradient, which is equivalent to solving a Poisson equation. The field is generally divided in sub-domains, based on the amplitude of the pressure gradient. The sequential pressure reconstruction is initiated from the outer domain, where the highest measurement accuracy is expected, hence where a reliable pressure reference can be taken. The pressure field in the other sub-domains is then computed by imposing Dirichlet boundary conditions stemming from the previously computed outer domain. Once the mean static pressure is reconstructed, the pressure integration constant $p_{B C}$ is adjusted so that the average of the reconstructed pressure computed along the contour of the PIV domain coincides with the average of the static pressure computed using the isentropic relation:

$$
p_{B C}=p_{\infty}\left[1+\frac{\gamma-1}{2} M_{\infty}^{2}\left(1-\frac{q^{2}}{u_{\infty}^{2}}\right)^{-\gamma /(\gamma-1)}\right]
$$

at the same location. The local stagnation pressure at the downstream plane eventually ensues from applying the local isentropic and isenthalpic relations to the previously determined local static pressure, following:

$$
p_{i}=p\left[\frac{1+\frac{\gamma-1}{2} M_{\infty}^{2}\left(1-\frac{q^{2}}{u_{\infty}^{2}}\right)}{1+\frac{\gamma-1}{2} M_{\infty}^{2}}\right]^{-\gamma /(\gamma-1)}
$$

A study was conducted on a model of the SACCON geometry, a generic flying wing configuration. The particular model used here has a wingspan $b=1 \mathrm{~m}$, a reference chord $c_{r e f}=0.31 \mathrm{~m}$ and a reference area $S_{\text {ref }}=0.3253 \mathrm{~m}^{2}$. It was placed inside the L1 wind-tunnel of the ONERA center of Lille. The freestream velocity was fixed at $u_{\infty}=35 \mathrm{~m} / \mathrm{s}$ throughout the campaign, which corresponds to a free-stream Reynolds number $R e=1.1 .10^{6}$, and a free-stream Mach number $M_{\infty}=0.10$. Three different angles of attack $\alpha=9^{\circ} ; 13^{\circ}$ and $18^{\circ}$ were investigated. The wake was measured one chord away from the model wingtips (Fig. 15 . The PIV images were processed using $32 \times 3$ pixels interrogation windows, with an overlap of $50 \%$, which resulted in velocity fields with a spatial resolution of $3 \mathrm{~mm}$. Time averages were performed over 5000 consecutive snapshots acquired at $5 \mathrm{~Hz}$ in order to guarantee reliable statistics. Finally, the longitudinal gradients present in equations were discretized by considering two additional planes, respectively located at $x=c_{\text {ref }}+/-30 \mathrm{~mm}$ (Fig. 15). Subsequently to stereo-PIV measurements, the left half of the model wake was surveyed using a five-hole probe. Fig. 16 presents the overall structure of the flow for $\alpha=13^{\circ}$.

The data of Fig. 17 strikingly show that the reconstructed static and stagnation pressure fields compare quite differently to their 5-hole probe counterparts. Indeed, the reconstructed static pressure is systematically lower than the direct probe measurements, while the reconstructed stagnation pressure appears to be much closer to the probe measurements, except for $\alpha=18^{\circ}$. Interestingly, the reconstructed stagnation pressure at low angles of attack appears to be quite accurate despite the fact that its computation uses a rather crude estimate of the static pressure field. This 
observation therefore suggests that the underestimation of the reconstructed static pressure is in fact mitigated by the velocity field, making the reconstructed stagnation pressure a more robust quantity for the purpose of wake integral methods.

The preliminary results shown here are promising, and suggest that stereo-PIV may indeed be a pertinent option when determining all far-field drag components acting on a model, within an industrial context [65]. First, the dramatic time savings introduced by this method makes it viable to measure a large number of samples. It is thus capable of yielding reliable flow statistics within a reasonable time-frame, unlike the five-hole probe method. This particular point may prove to be crucial when dealing with complex aerodynamic phenomena. Second, the fact that the relative error between the PIV predictions and the balance measurements is bounded is encouraging, and asks for a more thorough study of the conditions under which the Poisson equation for the static pressure is derived. All in all, the gains in accuracy and measuring time using the PIV method appear to compensate the extra work induced by the need to capture three PIV planes, and solve a Poisson equation.

\section{Conclusion}

This paper presents ONERA's post-processing development strategy that was initiated several years ago. This strategy has been motivated by advances in the fields of CFD and non-intrusive experimental measurement techniques. From numerical simulation data, the first post-processing codes and methods only determined the performance of aircraft based on the near-field approach, i.e. the integration of surface forces. The far-field approach developed and implemented at ONERA makes it possible to not only calculate the total drag force, but also break it down according to its different phenomenological sources. An additional advantage is its increased precision, giving the basis for an estimate of the quality of the results obtained by the calculation of spurious drag. The successful development and implementation of this approach has motivated its continuous pursuit, as well as the development of additional formulations dedicated to rotating flows and unsteady flows. This approach, which is now also the subject of work in other research centers, has led to a family of dedicated codes used at ONERA but also by aerospace manufacturers.

At the same time, evolutions and new constraints within the aviation world lead to the study of innovative concepts requiring both the improvement of our current tools but also the development of new ones. In particular, the concept of exergy gives the basis for an energetic analysis of numerical simulations complementary to the previous mechanical approach. On the other hand, the Lamb vector formulation is also a mechanical approach but based essentially on the velocity vector field and is different from the initial one. It aims to provide a better understanding of the links between aircraft performance and the vorticity field created.

Concerning the post-processing of experimental data, today's improvements concern the use of a less intrusive and faster wake survey measurement than the 5-hole probes used until now. In many research centers, PIV is already used and work in progress at ONERA should allow rapid progress in the extraction and breakdown of the drag force.

The following table presents the different approaches developed and the improvements in progress. Several research axes could be explored in the future: data assimilation and machine learning in particular could make it possible to establish a link between CFD and wind tunnel measurements to the benefit of both.

\begin{tabular}{lllll}
\hline \hline \multicolumn{2}{c}{ CFD } & & \multicolumn{2}{c}{ Wind tunnel } \\
\hline FFD & Lamb vector & Exergy & Five-hole probes & PIV \\
- steady/unsteady & $\bullet$ steady & $\bullet$ steady & $\bullet$ steady & ・ steady \\
- sub/transonic & $\bullet$ sub/transonic & $\bullet$ sub/transonic & $\bullet$ sub/transonic & $\bullet$ sub/transonic \\
- supersonic & & $\bullet$ supersonic & & \\
- power off/on & & $\bullet$ power off/on & \\
- rotating frame & & $\bullet$ rotating frame \\
- internal flow & & \\
\hline
\end{tabular}

Table 4 Overview of the domain of application of ONERA methods related to drag analysis and decomposition. Items currently under investigation are highlighted in blue. 


\section{References}

[1] Betz, A., "A Method for the Direct Determination of Wing-Section Drag," NACA TM-337, Reproduced from Zeitschrift für Flugtechnik und Motorluftschiffahrt, November 1925.

[2] Melville-Jones, B., "The measurement of profile drag by the pitot-traverse method, The Cambridge University Aeronautics Laboratory (R \& M No. 1688)," 1937.

[3] Oswatitsch, K., Gas Dynamics, Academic Press, New York, 1956.

[4] Maskell, E. C., "Progress Towards a Method for the Measurement of the Components of the Drag of a Wing of Finite Span," RAE Technical Report No. 72232, 1972.

[5] Kusunose, K., "Development of a universal wake survey data analysis code," 15th Applied Aerodynamics Conference, 1997, pp. AIAA Paper 97-2294.

[6] Kusunose, K., Crowder, J., and Watzlavick, R., "Wave drag extraction from profile drag based on a wake-integral method," 37th Aerospace Sciences Meeting and Exhibit, Reno, NV, USA, 1999, pp. AIAA Paper 1999-275. doi:10.2514/6.1999-275, URL https://arc.aiaa.org/doi/abs/10.2514/6.1999-275.

[7] Kusunose, K., "A wake integration method for airplane drag prediction," The 21st Century COE Program, International COE of flow Dynamics Lecture Series, Vol. 3, 2005.

[8] Steger, J. L., and Baldwin, B. S., "Shock Waves and Drag in the Numerical Calculation of Isentropic Transonic Flow," NASA Technical Note D-6997, 1972.

[9] Henne, P. A., and Hicks, R. M., "Wing analysis using a transonic potential flow computational method," NASA Technical Memorandum 78464, 1978.

[10] Yu, N. J., Chen, H. C., Samant, S. S., and Rubbert, P. E., "Inviscid drag calculations for transonic flows," 6th Computational Fluid Dynamics Conference Danvers, July 1983, pp. AIAA Paper 1983-1928.

[11] Lock, R. C., "Prediction of the Drag of Wongs at Subsonic Speeds by Viscous/Inviscid Interaction Technics," Aircraft Drag Prediction and Reduction, 1985, pp. AGARD-R-723.

[12] Rizzi, A., "Spurious entropy production and very accurate solutions to the Euler equations," The Aeronautical Journal, Vol. 89, No. 882, 1985, pp. 59-71.

[13] Destarac, D., "Far-Field Drag in Transonic Potential Flow: Analysis and Optimisation," Proceedings of the 1993 European Forum, Recent Developments and Applications in Aeronautical CFD, The Royal Aeronautical Society, Bristol, September 1993.

[14] van Dam, C. P., Nikfetrat, K., Chang, I. C., and Vijgen, P. M. H. W., "Drag calculations of wings using Euler methods," 29th Aerospace Sciences Meeting, January 1991, pp. AIAA Paper 91-0338. doi:10.2514/6.1991-338, URL https://arc . aiaa. org/doi/abs/10.2514/6.1991-338.

[15] van Dam, C. P., and Nikfetrat, K., "Accurate prediction of drag using Euler methods," Journal of Aircraft, Vol. 29, No. 3, May-June 1992, pp. 516-519. doi:10.2514/3.46194, URL https://doi.org/10.2514/3.46194

[16] van der Vooren, J., and Slooff, J. W., "CFD-Based Drag Prediction: State-of-the-Art, Theory, Prospects," Lecture Notes, AIAA Professional Studies, Course on Drag Prediction and Measurment, 1990.

[17] Tognaccini, R., and Paparone, L., "Drag Breakdown aand Thrust-Drag Bookkeeping from CFD Calculations," Proceedings of the Workshop on Aerodynamic Engine/Aircraft Integration For Transport Aircraft, Braunschweig, 2000.

[18] van der Vooren, J., and Destarac, D., "Drag/thrust analysis of jet-propelled transonic transport aircraft; definition of physical drag components," Aerospace Science and Technology, Vol. 8, No. 6, 2004, pp. 545-556. doi:https://doi.org/10.1016/j.ast.2004. 03.004 .

[19] Destarac, D., Far-Field / Near-Field Drag Balance and Applications of Drag Extraction in CFD, In: CFD-based Aircraft Drag Prediction and Reduction, VKI Lecture Series 2003, Von Karman Institute for Fluid Dynamics, Rhode Saint Genèse, February 3-7, 2003, National Institute of Aerospace, Hampton (VA), November 3-7, 2003.

[20] Destarac, D., "Spurious far-field-boundary induced drag in two-dimensional flow simulations," Journal of Aircraft, Vol. 48, No. 4, 2011, pp. 1444-1455. doi:http://dx.doi.org/10.2514/1.C031331. 
[21] Destarac, D., "Investigating Negative Drag in Grid Convergence for Two-Dimensional Euler Solutions," Journal of Aircraft, Vol. 48, No. 4, 2011, pp. 1468-1470. doi:http://dx.doi.org/10.2514/1.C031330.

[22] Destarac, D., "Three-Component Breakdown of Spurious Drag in Computational Fluid Dynamics," Journal of Aircraft, Vol. 52, No. 4, 2015, pp. 1336-1344. doi:10.2514/1.C032924.

[23] Verley, S., "Evaluation du couple "champ lointain" d'un rotor d'hélicoptère en vol stationnaire: analyse de résultats issus de simulations numériques de mécanique des fluides," Ph.D. thesis, Université d'Orléans, 2012.

[24] Verley, S., "Torque Analysis based on the CFD "Far-field" Philosophy for Rotors in Hover," 46th 3AF Symposium of Applied Aerodynamis, Orléans, France, 28-30 March, 2011.

[25] Méheut, M., "Thrust and Torque Far-field Analysis of Propeller and Counter Rotating Open Rotor Configurations," 31 st AIAA Applied Aerodynamics Conference, Fluid Dynamics and Co-located Conferences, AIAA Paper 2013-2803, American Institute of Aeronautics and Astronautics, 2013. doi:10.2514/6.2013-2803, URL https://doi .org/10.2514/6.2013-2803

[26] Cambier, L., Heib, S., and Plot, S., “The Onera elsA CFD software: input from research and feedback from industry," Mechanics \& Industry, Vol. 14, No. 3, 2013, pp. 159-174.

[27] Gariepy, M., Trepanier, J.-Y., and Malouin, B., "Generalization of the far-field drag decomposition method to unsteady flows," AIAA Journal, Vol. 51, No. 6, 2013, pp. 1309-1319.

[28] Toubin, H., and Bailly, D., "Far-field drag decomposition for unsteady flows," Proceedings of 49th International Symposium of Applied Aerodynamics, Lille, France, 2014.

[29] Toubin, H., Bailly, D., and Costes, M., "Improved Unsteady Far-Field Drag Breakdown Method and Application to Complex Cases," AIAA Journal, Vol. 54, No. 6, 2016, pp. 1907-1921. doi:10.2514/1.J054756, URL https://doi.org/10.2514/1. JQ54756

[30] Toubin, H., Bailly, D., and Costes, M., "Improvements in the New Unsteady Far-Field Drag Breakdown Method and Application to Complex Cases," 33rd AIAA Applied Aerodynamics Conference, Paper 2015-2877, American Institute of Aeronautics and Astronautics, 2015.

[31] Toubin, H., "Prediction and phenomenological breakdown of drag for unsteady flows," Ph.D. thesis, Université Pierre et Marie Curie de Paris VI, 2015.

[32] Deck, S., "Numerical Simulation of Transonic Buffet over a Supercritical Airfoil," AIAA Journal, Vol. 45, No. 7, 2005 , pp. $1556-1566$.

[33] Hue, D., Chanzy, Q., and Landier, S., "DPW-6: Drag Analyses and Increments Using Different Geometries of the Common Research Model Airliner," Journal of Aircraft, Vol. 55, No. 4, 2018, pp. 1509-1521. doi:10.2514/1.C034139, URL https://doi.org/10.2514/1.C034139

[34] Hue, D., Vermeersch, O., Bailly, D., Brunet, V., and Forte, M., "Experimental and Numerical Methods for Transition and Drag Predictions of Laminar Airfoils," AIAA Journal, Vol. 53, No. 9, 2015, pp. 2694-2712. doi:10.2514/1.J053788, URL https://doi.org/10.2514/1.J053788

[35] Hue, D., "Fifth Drag Prediction Workshop: ONERA Investigations with Experimental Wing Twist and Laminarity," Journal of Aircraft, Vol. 51, No. 4, 2014, pp. 1311-1322. doi:10.2514/1.C032438, URL https://doi .org/10.2514/1.C032438

[36] Hantrais-Gervois, J.-L., and Destarac, D., "Drag Polar Invariance with Flexibility,” Journal of Aircraft, Vol. 52, No. 3, 2015, pp. 997-1001. doi:10.2514/1.C033193, URL https://doi.org/10.2514/1.CQ33193

[37] Hue, D., "Fifth Drag Prediction Workshop: Computational Fluid Dynamics Studies Carried Out at ONERA," Journal of Aircraft, Vol. 51, No. 4, 2014, pp. 1295-1310. doi:10.2514/1.C032054, URL https://doi .org/10.2514/1.C032054

[38] Ghazlane, I., "Adjoint-based aerostructural sensitivity analysis for wing design,” Ph.D. thesis, Université de Nice-Sophia Antipolis, 2012.

[39] Salah El Din, I., Dumont, A., and Blondeau, C., "Transonic Wing-body Civil Transport Aircraft Aero-Structural Design Optimization using a Bi-Level High Fidelity Approach - A Focus on the Aerodynamic Process," 51st AIAA Aerospace Sciences Meeting including the New Horizons Forum and Aerospace Exposition, AIAA Paper 2013-0144, 2013. 
[40] Carrier, G., Destarac, D., Dumont, A., Méheut, M., Salah El Din, I., Peter, J., Ben Khelil, S., Brézillon, J., and Pestana, M., "Gradient-Based Aerodynamic Optimization with the elsA Software," 52nd AIAA Aerospace Sciences Meeting, AIAA Paper 2014-0568, 2014.

[41] Méheut, M., Arntz, A., and Carrier, G., "Aerodynamic Shape Optimizations of a Blended Wing Body Configuration for Several Wing Planforms," 30th AIAA Applied Aerodynamics Conference, AIAA Paper 2012-3122, American Institute of Aeronautics and Astronautics, 2012. doi:10.2514/6.2012-3122, URL https://doi .org/10.2514/6.2012-3122

[42] Carini, M., Méheut, M., Kanellopoulos, S., Cipolla, V., and Abu Salem, K., "Aerodynamic analysis and optimization of a boxwing architecture for commercial airplanes," AIAA Scitech 2020 Forum, Paper 2020-1285, 2020.

[43] Wiart, L., Atinault, O., Grenon, R., Paluch, B., and Hue, D., "Development of NOVA Aircraft Configurations for Large Engine Integration Studies," 33rd AIAA Applied Aerodynamics Conference, American Institute of Aeronautics and Astronautics, 2015.

[44] Wu, J. Z., Ma, H. Y., and Zhou, M. D., Vorticity and vortex dynamics, Springer Science \& Business Media, 2007.

[45] Ueno, M., Tognaccini, R., and Marongiu, C., "Lift-Induced Drag Computation by Lamb Vector Integration," 40th Fluid Dynamics Conference and Exhibit, Paper 2010-5094, American Institute of Aeronautics and Astronautics, 2010.

[46] Marongiu, C., Tognaccini, R., and Ueno, M., "Lift and lift-induced drag computation by Lamb vector integration," AIAA Journal, Vol. 51, No. 6, 2013, pp. 1420-1430. doi:10.2514/1.J052104.

[47] Mele, B., and Tognaccini, R., "Aerodynamic force by Lamb vector integrals in compressible flow," Physics of Fluids, Vol. 26, No. 5, 2014, p. 056104. doi:10.1063/1.4875015.

[48] Prandtl, L., “Theory of lifting surfaces,” Tech. Rep. NACA TN10, 1919.

[49] Yang, Y. T., Zhang, R. K., An, Y. R., and Wu, J. Z., "Steady vortex force theory and slender-wing flow diagnosis," Acta Mechanica Sinica, Vol. 23, No. 6, 2007, pp. 609-619. doi:10.1007/s10409-007-0107-0.

[50] Fournis, C., Bailly, D., and Tognaccini, R., "A reference point invariant Lamb vector based aerodynamic force breakdown in steady compressible flows," AIAA Scitech 2020 Forum, Paper 2020-1996, 2020. doi:10.2514/6.2020-1996, URL https: //arc.aiaa.org/doi/abs/10.2514/6.2020-1996

[51] Fournis, C., Bailly, D., and Tognaccini, R., "Definition of an Invariant Lamb-Vector-Based Aerodynamic Force Breakdown Using Far-Field Flow Symmetries,” AIAA Journal, Vol. 59, No. 1, 2021, pp. 34-48. doi:10.2514/1.J059591.

[52] Fournis, C., Bailly, D., and Tognaccini, R., "Compressibility Correction to Kutta-Joukowski and Maskell Formulas Using Vortex-Force Theory,” AIAA Journal, Vol. 59, No. 2, 2021, pp. 758-763. doi:10.2514/1.J059710.

[53] Fournis, C., Bailly, D., and Tognaccini, R., "An invariant vortex-force theory related to classical far-field analyses in transonic flows," AIAA Aviation 2021 Forum, 2021.

[54] Schmitz, S., "Finite Domain Viscous Correction to the Kutta-Joukowski Theorem in Incompressible Flow," AIAA Journal, Vol. 52, No. 9, 2014, pp. 2079-2083. doi:10.2514/1.J053114.

[55] Drela, M., "Power balance in aerodynamic flows," AIAA Journal, Vol. 47, No. 7, 2009, pp. 1761-1771.

[56] Arntz, A., "Civil Aircraft Aero-thermo-propulsive Performance Assessment by an Exergy Analysis of High-fidelity CFD-RANS Flow Solutions,” Ph.D. thesis, Lille 1 Université - Sciences et Technologies, Lille, France, 2014.

[57] Arntz, A., and Atinault, O., "Exergy-Based Performance Assessment of a Blended Wing-Body with Boundary-Layer Ingestion," AIAA Journal, Vol. 53, No. 12, 2015, pp. 3766-3776.

[58] Arntz, A., Atinault, O., and Merlen, A., "Exergy-Based Formulation for Aircraft Aeropropulsive Performance Assessment: Theoretical Development," AIAA Journal, Vol. 53, No. 6, 2015, pp. 1627-1639.

[59] Arntz, A., and Hue, D., "Exergy-Based Performance Assessment of the NASA Common Research Model," AIAA Journal, Vol. 54, No. 1, 2016, pp. 88-100.

[60] Petropoulos, I., Wervaecke, C., Bailly, D., and Derweduwen, T., "Numerical investigations of the exergy balance method for aerodynamic performance evaluation," AIAA Aviation Forum, Paper 2019-2926, American Institute of Aeronautics and Astronautics, 2019. doi:10.2514/6.2019-2926, URL https://doi .org/10.2514/6.2019-2926 
[61] Wervaecke, C., Petropoulos, I., and Bailly, D., Assessment of Exergy Analysis of CFD Simulations for the Evaluation of Aero-Thermo-Propulsive Performance of Aerial Vehicles, Springer International Publishing, Cham, 2021, pp. $261-275$. doi:10.1007/978-3-030-57422-2_17, URL https://doi.org/10.1007/978-3-030-57422-2_17.

[62] Aguirre, M. A., and Duplaa, S., "Exergetic Drag Characteristic Curves,” AIAA Journal, Vol. 57, No. 7, 2019 , pp. $2746-2757$.

[63] Wiart, L., and Negulescu, C., "Exploration of the Airbus "NAUTILIUS" engine integration concept," 31st Congress of the International Council of the Aeronautical Sciences (ICAS), Belo Horizonte, Brazil, September 2018.

[64] Méheut, M., and Bailly, D., "Drag Breakdown Methods From Wake Measurements." AIAA Journal, Vol. 46, No. 4, 2008 , pp. 847-862. doi:10.2514/1.29051, URL/https://arc.aiaa.org/doi/10.2514/1.29051

[65] Baker, N., Diaz, D., Bailly, D., David, L., and Monnier, J.-C., "Mechanical vs. phenomenological formulations to determine mean aerodynamic drag from stereo-PIV wake measurements," Experiments in Fluids, Vol. 60, No. 167, 2019. 10.1007/s00348-019-2813-3, URL https://doi.org/10.1007/s00348-019-2813-3.

[66] Jeon, Y., Gomit, G., Earl, T., Chatellier, L., and David, L., "Sequential least-square reconstruction of instantaneous pressure field around a body from TR-PIV," Experiments in Fluids, Vol. 59, 2018. 